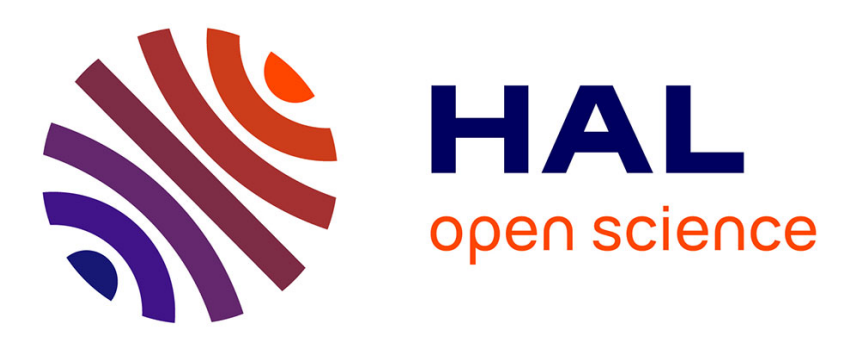

\title{
Non linear elasticity, viscosity and damage in open-cell polymeric foams
}

Giampiero Pampolini, Michel Raous

\section{To cite this version:}

Giampiero Pampolini, Michel Raous. Non linear elasticity, viscosity and damage in open-cell polymeric foams. Archive of Applied Mechanics, 2014, 84 (12), pp.1861-1881. 10.1007/s00419-014-0891-5 . hal01353394

\section{HAL Id: hal-01353394 \\ https://hal.science/hal-01353394}

Submitted on 11 Aug 2016

HAL is a multi-disciplinary open access archive for the deposit and dissemination of scientific research documents, whether they are published or not. The documents may come from teaching and research institutions in France or abroad, or from public or private research centers.
L'archive ouverte pluridisciplinaire HAL, est destinée au dépôt et à la diffusion de documents scientifiques de niveau recherche, publiés ou non, émanant des établissements d'enseignement et de recherche français ou étrangers, des laboratoires publics ou privés. 


\title{
Non linear elasticity, viscosity and damage in open-cell polymeric foams
}

Received: date / Accepted: date

\begin{abstract}
Of interest in this work is the behavior of open-cell polymer foams under compression. In a recent work by Del Piero and Pampolini (2012) a model coupling non linear elasticity and viscosity was introduced to describe the response of a polyurethane foam subjected to uniaxial cyclic compression. Non elastic effects of the response curves were attributed to the viscous properties of the foam, while strain localization and hysterisis were attributed to the non convexity of the strain energy density. But the model could not reproduce the response curves after the first loadingunloading cycle. Here that model is extended by taking into account the damage of the foam. A simple phenomenological one parameter damage law is used to describe the damage of the cell walls occurring during the first loading cycle. An accurate identification procedure for the model constants is also developed and the mutual role played by viscosity and damage on the foam deformation evolution is discussed. Besides the one dimensional formulation, the model permits a precise analysis of the main phenomena which have to be considered to describe the complex behavior of the foam.
\end{abstract}

Keywords Polymeric foams · cyclic behavior · non convex energy $\cdot$ linear viscosity $\cdot$ damage Mullins effect

\section{Introduction}

Open-cell polymer foams are widely used in engineering systems because of their specific mechanical properties. Their applications include energy absorbing components, seat cushions, packaging material and lightweight composite sandwich structures. A complete review of the properties of various types of foams can be found in the book of Gibson and Ashby [7].

The mechanical behavior of foam is basically studied under monotonic compression. In compression experiments $[10,29,31,32,34]$, various relevant phenomena were observed: the deformations were localized in bands orthogonal to the loading direction and the response curve showed a large hysteresis loop, rate dependence, and a softening phenomenon that consists in a gradual decrease of strength during the cyclic loading.

With a view to relate the mechanical properties of foams to the characteristics of the microstructure, many authors introduced microstructural models (e.g. see $[9,20,30,33])$ in which the foam is represented as a periodic structure based on the repetition of a basic cell composed of linear elastic beams. Various basic cells as cubes [7], tetrahedrons [33] or dodecahedrons [17] were used. In the

G. Pampolini

Laboratoire de Mécanique et d'Acoustique, CNRS, 31 chemin Joseph Aiguier 13402 Marseille, France E-mail: pampolini@lma.cnrs-mrs.fr

Present address: Saint-Gobain Recherche, 39 quai Lucien Lefranc, 93303 Aubervilliers, France of G. Pampolini

M. Raous

Laboratoire de Mécanique et d'Acoustique, CNRS, 31 chemin Joseph Aiguier 13402 Marseille, France 
work by Gong and Kyriakides [9] the foam was modeled as a periodic structure of Kelvin cells elongated in one direction and composed of shear-deformable linear elastic beams with a non-uniform cross sectional area. It was shown that the basic cell has to accurately reproduce the characteristics and the geometry of the microstructure to give a good description of the mechanical behavior of the foams. X-ray tomography analysis [15] and Surface Evolver softwares [20] can possibly be used to obtain more information about the microstructure and thus generate more realistic models. These studies based on microstructural analyses established that the basic mechanical process giving rise to strain localization results from the buckling of the cell struts. Significant insights into this behavior were obtained in [11], where various post-buckling responses associated with deformation modes with long wavelengths involving a group of cells or wavelengths of the order of the cell dimension, were obtained under various loading conditions.

Developing a complete numerical simulation of a foam microscale deformation process is a challenging task [1], due to the complex microstructural geometry, the extremely large deformations, and the contact which occurs between the collapsed struts. It is also difficult to include rate dependence and stress softening effects in microstructural models.

In a different approach, strain localization and hysteresis were described in the context of nonlinear elasticity by assuming a non convex strain energy density $[8,24]$. The non-convexity of the strain energy induces non uniqueness of equilibrium configurations under a given load. These configurations can be associated to different phases of the material [6]. Extensions of these models including the rate-dependent properties of foams have been presented in $[3,27]$. In particular, in the former work the foam was represented as a chain, each element of which consisted of a non-linear elastic spring connected in parallel to a linear visco-elastic element. The rheological model was completed by adding a visco-elastic element set in parallel with the chain. But with that model major discrepancies still remained between theory and experiments when considering the difference of the response curves between the first and subsequent cycles.

The softening phenomenon observed in foams is reminiscent of the Mullins effect occurring in filler-reinforced rubbers [21]. In the literature alternative models describing the stress softening effect occurring in rubbers as a form of damage have been proposed (e.g. see $[2,4,5,19,22])$. This interpretation is used here for open cell foams, and the stress softening is attributed to damage of the foam cell walls during first compression. The first contribution of the present study is to refine the rheological model proposed by Del Piero and Pampolini [3] by introducing damage. The non linear elastic springs are considered as damageable elements and extra damage variables and their work-conjugated forces are introduced. Evolution of damage is controlled by only one parameter. In spite of its simplicity the model gives a good picture of the transition between first and second cycle.

Due to the complex behavior of foams, the complete model requires a large number of material constants to be experimentally identified. The second contribution of the present study is the construction of an identification process that combines qualitative analysis with optimization methods. The identification process here developed have permitted the numerical simulations carried out simultaneously with the present work in [3]. The validity of the model is evaluated by comparing numerical simulation results and experimental data for a set of complex loading cycles.

In Section 2, we review some experimental results, showing that reproducing the experimental response needs to include non linear elasticity, viscosity and damage. In Section 3, the model is constructed stepwise. The model based on the use of non linear elastic springs introduced in [24] is first briefly recalled. The viscoelastic elements developed by Del Piero and Pampolini [3] are added, and the damage is then included. Section 4 deals with the numerical implementation of the model, Section 5 describes the procedure developed to identify the material constants, and the numerical simulation results are discussed and compared with various experiments in Section 6.

\section{Response to uniaxial compression cycles}

The experimental force/elongation curves of open-cell polymer foams subjected to compression loads show a characteristic path composed of a first almost linear branch, followed by a long plateau and a second ascending branch. During the plateau regime, deformation localized in bands orthogonal to the loading directions is observed $[24,31,32]$. This localization phenomenon is due to the gradual buckling of the cell walls. When all the cell walls have collapsed, the foam is compacted and the 
(a)

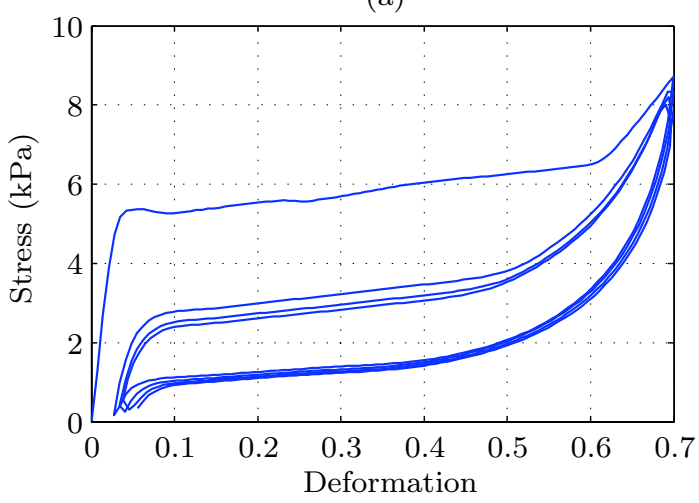

(b)

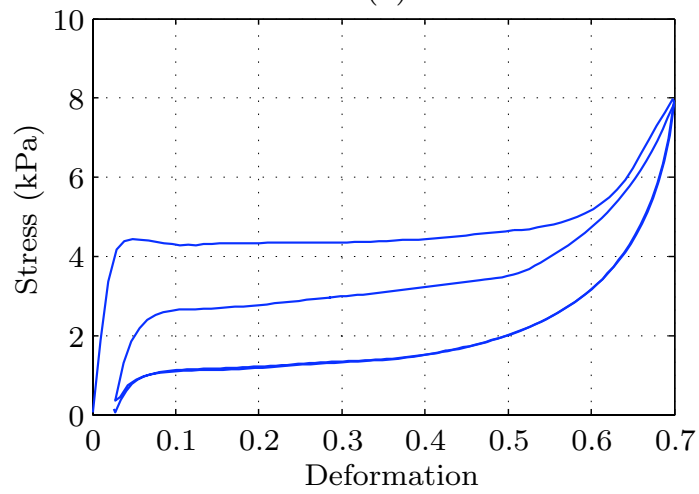

(c)

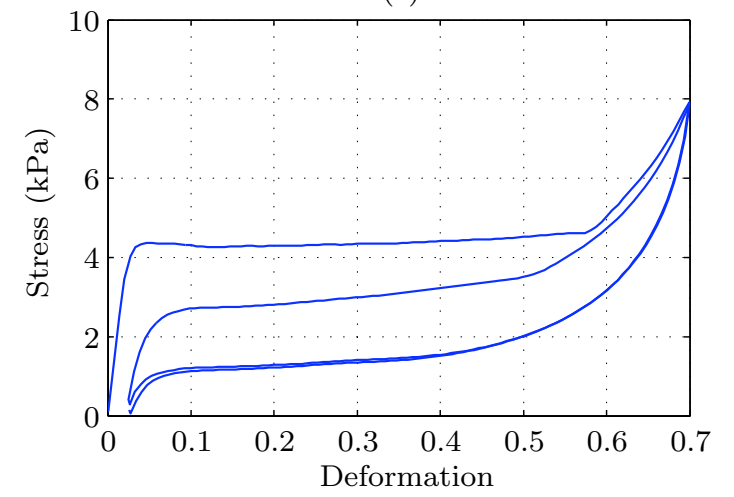

Fig. 1 Cyclic test after resting periods. Force versus displacement curves obtained on a virgin sample (a) and on the same sample after resting periods of 2 days (b) and 17 days (c) (velocity $5 \mathrm{~mm} / \mathrm{min}$ )

resistance to further compression increases, resulting in the second ascending branch of the response curve. At unloading, instead of following the initial loading path the response describes a large hysteresis loop.

An increase in the loading velocity results in an overall upward shift of the response curves. This effect is more pronounced during loading than at unloading and the residual deformation is found to be almost independent of the loading velocity [34]. The viscous properties of the foam play a significant role even in the response at small amplitude cyclic tests. In the work by Del Piero and Pampolini [3] small amplitude loading-unloading cycles with intermittent holding periods were carried out on open-cell polyurethane foam. The occurrence of stress relaxation effects corresponding to the holding periods confirmed the viscous behavior of the foam.

On the contrary, the significant strength reduction effects observed in high amplitude cyclic compression tests can not be entirely attributed to the viscous properties of the foam. To clarify this point, cyclic compression experiments on open-cell polyurethane foams ${ }^{1}$ were performed. Most of the experiments presented here were available in $[3,23,24]$. They are re-interpreted in the present work since the previous model were unsatisfactory.

Figure 1a shows the response curves ${ }^{2}$ to four high amplitude loading-unloading cycles made on a virgin sample. Note that the strength reduction, which is significant between the first and second cycles, becomes negligible after five or six cycles (see also [10]). The initial strength is not fully recovered after few days of rest. In particular, by comparing the response curves on the virgin specimen (Figure 1a) and those recorded after a two-day rest (Figure 1b) it can be observed that

\footnotetext{
1 All experiments were conducted on a open-cell polyether urethane foam with a density of $27 \mathrm{~kg} / \mathrm{m}^{3}$ and manufactured by Chemical Resine SAS (Lissone, Italy).

2 In all figures, Deformation denotes the prescribed displacement divided by the initial thickness of the sample, and Stress denotes the measured force divided by the initial cross-section area of the sample. Compression deformation and force are taken to be positive.
} 
after two-day of rest the loading curve of the first cycle reaches the plateau at a lower value of the force. However, we observe that the curves of the second cycle in Figure 1b almost coincide with the third-cycle curves obtained on the virgin sample. This means that only the first cycle made on the virgin sample causes a permanent strength reduction. After a further 17-day rest (Figure 1c), no significant changes in the response curves were observed.

In the model presented below, the strength reduction is attributed to damage.

\section{The model}

\subsection{The non linear elastic model}

In this section we briefly recall the purely non linear elastic model by Pampolini and Del Piero [24], where both strain localization and hysteresis are described in the context of non linear elasticity.

The foam is divided in horizontal layers of cells and each layer is represented as a non linear elastic spring, so that the entire foam is represented as a chain of $N$ springs. Each spring has a non convex (double well) strain energy density, and this implies that, for the force in a specific range of values, each spring has two stable equilibrium configurations corresponding to two phases of the material. The two phases stand for the stages in the foam which occur before the cell walls collapse, when voids are present, and after the walls have collapsed, when the cell is compacted.

Let us consider the chain subjected to the hard device condition

$$
\sum_{i=1}^{N} \varepsilon_{i}=N \varepsilon_{0},
$$

where $\varepsilon_{i}$ is the deformation of the $i$-th spring and $\varepsilon_{0}$ is the total prescribed deformation. The basis are rigid and the horizontal friction effects are negligable. Assume that all the springs have the non convex strain energy density $w[24]$

$$
\begin{gathered}
w\left(\varepsilon_{i}\right)=w_{a}\left(\varepsilon_{i}\right)+w_{b}\left(\varepsilon_{i}\right), \\
w_{a}\left(\varepsilon_{i}\right)=c\left(1+\varepsilon_{i}\right)^{m}\left(\frac{1}{m+2}\left(1+\varepsilon_{i}\right)^{2}-\frac{1}{m}\right)+\gamma_{a}, \\
w_{b}\left(\varepsilon_{i}\right)=\frac{1}{2} \alpha\left(1+\varepsilon_{i}\right)^{2}-\mu \log \left(1+\varepsilon_{i}\right)+\frac{\beta \sqrt{\pi}}{2 \sqrt{k}} \operatorname{erf}\left(\sqrt{k}\left(1+\varepsilon_{i}-a\right)\right)+\gamma_{b},
\end{gathered}
$$

where $\operatorname{erf}(\cdot)$ is the error function ${ }^{3}$ and $c, m, \mu, \beta, k, a$ are positive constants, which role will be discussed in Section 5, and the constants

$$
\begin{gathered}
\alpha=\mu-\beta \exp \left(-k(a-1)^{2}\right), \\
\gamma_{a}=\frac{2 c}{m(m+2)}, \\
\gamma_{b}=-\frac{1}{2}\left(\mu-\beta \exp \left(-k(a-1)^{2}\right)\right)-\frac{\beta \sqrt{\pi}}{2 \sqrt{k}} \operatorname{erf}(\sqrt{k}(1-a)),
\end{gathered}
$$

are determined by the condition that both the strain energy and the force must be equal to zero in the reference configuration $\varepsilon=0$. Note that the strain energy is composed of two terms: $w_{a}$ that describes the behavior of the foam cell at low values of deformation, before the collapse of the cell walls, and $w_{b}$ that describes the behavior at high values of deformation when the cell is compacted.

The expression for the force

$$
\begin{gathered}
\sigma_{i}^{e}\left(\varepsilon_{i}\right)=\sigma_{a}\left(\varepsilon_{i}\right)+\sigma_{b}\left(\varepsilon_{i}\right), \\
\sigma_{a}\left(\varepsilon_{i}\right)=c\left(1+\varepsilon_{i}\right)^{m-1}\left(\left(1+\varepsilon_{i}\right)^{2}-1\right), \\
\sigma_{b}\left(\varepsilon_{i}\right)=\left(\mu-\beta \exp \left(-k(a-1)^{2}\right)\right)\left(1+\varepsilon_{i}\right)-\frac{\mu}{1+\varepsilon_{i}}+\beta \exp \left(-k\left(1+\varepsilon_{i}-a\right)^{2}\right),
\end{gathered}
$$

is obtained by differentiation of Eq. (2). The graphs showing the energy $w$ and the force $\sigma$ versus the deformation $\varepsilon$ are given in Figures $2 \mathrm{a}$ and $2 \mathrm{~b}$, respectively.

\footnotetext{
3 The error function is defined as follows: $\operatorname{erf}(x)=2 /(\sqrt{\pi}) \int_{0}^{x} \exp \left(-t^{2}\right) d t$
} 

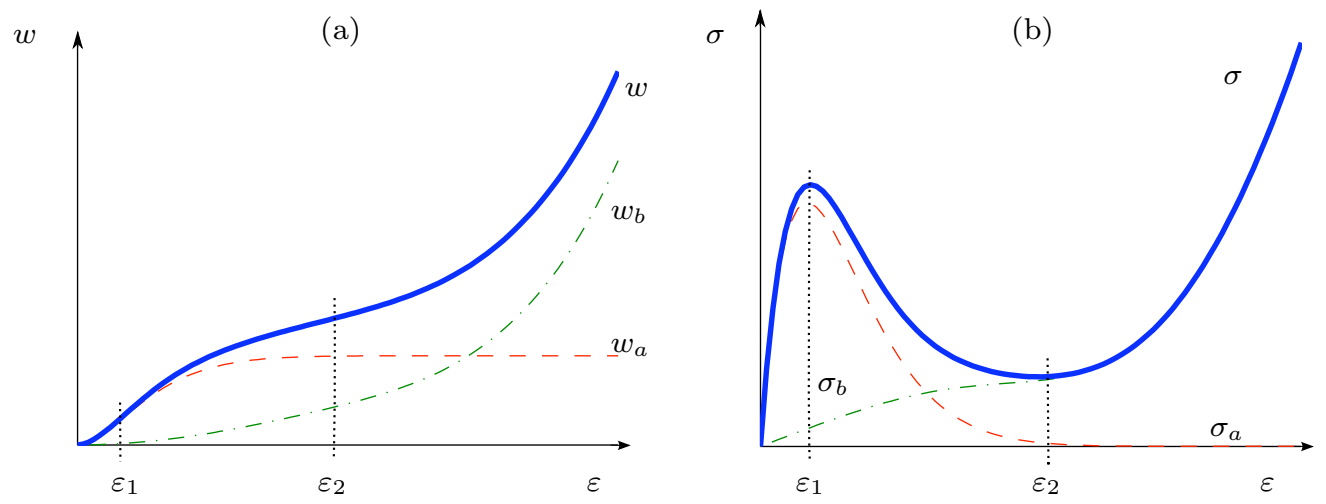

Fig. 2 Graph of the energy of a single non linear spring (a) and that of the force $\sigma$ versus the deformation $\varepsilon$ (b).

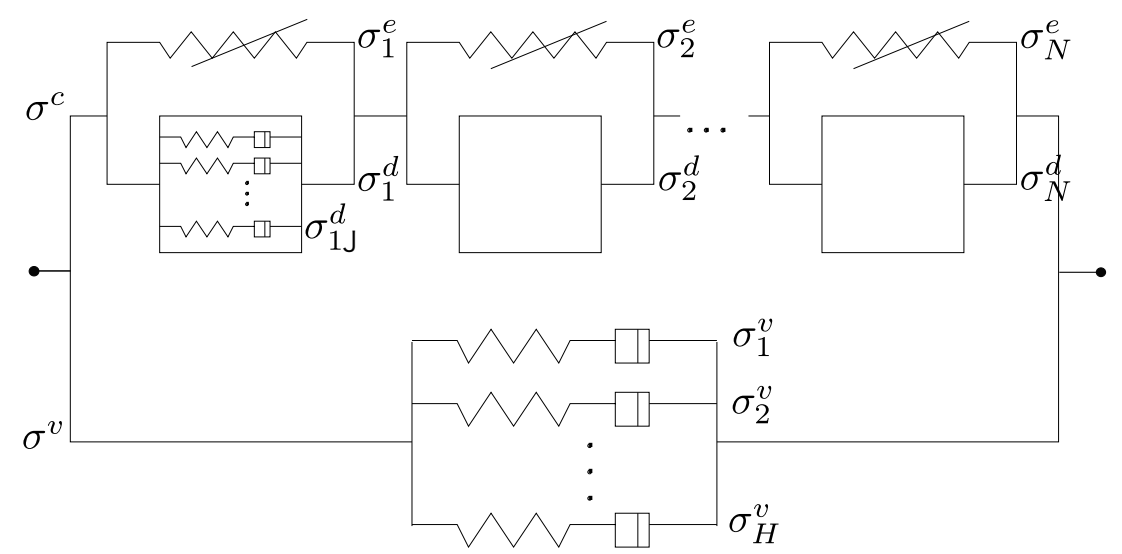

Fig. 3 The model.

\subsection{The viscoelastic model}

During the identification process, the analysis of the experiments showed the need of distinguishing the overall viscosity of the foam from that of the cells. Viscous effects are included in the model by adding two dissipative elements to the chain of the springs: one element is added to each spring in the chain, and another one is connected in parallel to the whole chain. From the mechanical viewpoint, the internal dissipative elements reproduce the viscous properties of the cells while the external ones reproduce the viscous properties of the bulk foam.

The model is presented in a general formulation in [3], based on a constitutive law for the dissipative elements of the Boltzmann Volterra type. Here the single internal and external dissipative elements are replaced by respectively $J$ and $H$ Maxwell elements set in parallel, see Figure 3 .

Let us consider the system subjected to the hard device condition (1). We take $\sigma_{i j}^{d}$ to denote the force in the $j$-th Maxwell element of the $i$-th internal dissipative element, and $\sigma_{h}^{v}$ to denote the force in the $h$-th Maxwell element of the external dissipative element. The total forces in the internal and external dissipative elements are denoted by $\sigma_{i}^{d}$ and $\sigma^{v}$, respectively. The constitutive laws for the dissipative elements can be written as follows:

$$
\begin{array}{ll}
\sigma_{i}^{d}=\sum_{j=1}^{J} \sigma_{i j}^{d}, & \sigma^{v}=\sum_{h=1}^{H} \sigma_{h}^{v}, \\
\dot{\sigma}_{i j}^{d}+p_{j} \sigma_{i j}^{d}=k_{j} \dot{\varepsilon}_{i}, & \dot{\sigma}_{h}^{v}+p_{h} \sigma_{h}^{v}=k_{h} \dot{\varepsilon}_{0},
\end{array}
$$

where $k_{j}, k_{h}, p_{j}, p_{h}$ are positive constants, and the dot denotes time differentiation. 
The total force $\sigma$ is the sum of the force $\sigma^{c}$ acting on the chain and the force $\sigma^{v}$ acting on the single external dissipative element

$$
\sigma=\sigma^{c}+\sigma^{v}
$$

and since the force $\sigma^{c}$ is the sum $\sigma_{i}^{e}+\sigma_{i}^{d}$ for all the elements, we have

$$
\sigma=\sigma_{i}^{e}+\sigma_{i}^{d}+\sigma^{v} \quad i \in\{1, \ldots N\}
$$

\subsection{The viscoelastic model with damage}

The experiments in cyclic compression presented in Section 2 show that a strength reduction in the response curve at loading is observed after the first cycle and that this strength reduction is not recovered after some days of rest. This effect is here attributed to a damage phenomenum. Damage occurs in the first loading process during progressive collapse of the cell. Once the cell walls are damaged, a lower force suffices to crush the cells and a lower stress level is therefore reached at the plateau in the subsequent loading cycles.

This idea is introduced into the model by taking the non linear elastic springs to be damageable elements. As described in Section 3.1, the strain energy density of the springs is the sum of the two terms $w_{a}$ and $w_{b}$ that characterize the behavior at low and high deformations, respectively. According to continuum damage mechanics theory (e.g. see [18]), we can express the strain energy density of the $i$-th damageable non linear spring in term of the undamaged strain energy densities $w_{a}$ and $w_{b}$ as follows:

$$
w\left(\varepsilon_{i}, d_{i}\right)=\left(1-d_{i}\right) w_{a}\left(\varepsilon_{i}\right)+w_{b}\left(\varepsilon_{i}\right)
$$

where the internal variable $d_{i} \in[0,1]$ is a scalar referred as the damage variable and $\left(1-d_{i}\right)$ is a reduction factor which has been suggested by Kachanov [16]. Note that in Eq. (8), we take the reduction factor to affect only $w_{a}$. This simplification is motivated by the results of the experiments shown in Figure 1, where the main differences in the response curves between the first and the subsequent cycles concern the behavior before cell wall collapse and the stress level reached in the plateau.

By differentiation of the strain energy density with respect to the deformation $\varepsilon_{i}$ and the damage variable $d_{i}$, one obtains the expression of the force $\sigma_{i}^{e}$ and of the thermodynamic force $f_{i}$ workconjugated to the damage variable, respectively

$$
\begin{aligned}
\sigma_{i}^{e} & =\frac{\partial w\left(\varepsilon_{i}, d_{i}\right)}{\partial \varepsilon_{i}}=\left(\left(1-d_{i}\right) \sigma_{a}\left(\varepsilon_{i}\right)+\sigma_{b}\left(\varepsilon_{i}\right)\right) . \\
f_{i} & =-\frac{\partial w\left(\varepsilon_{i}, d_{i}\right)}{\partial d_{i}}=w_{a}\left(\varepsilon_{i}\right)
\end{aligned}
$$

Note in Eq. (9)a that if the spring is fully damaged $(d=1)$, the contribution of $\sigma_{a}$ vanishes and the spring offers low resistance to initial crushing but still the same resistance to large compression.

The softening effect described in Section 2 is reminiscent of the Mullins effect observed in filled reinforced rubber. A large number of models have been proposed in the literature to describe the Mullins effect as a form of damage with different assumptions for the damage law and the damage criterion, see [4]. In one simple class of models based on the works by Gurtin and Francis [12] and Simo [28], damage is assumed to be function of the maximal deformation attained over the past history. Here we adapt this idea for open-cell polymer foams, and we define the following damage criterion

$$
\phi_{i}=\varepsilon_{i}(t)-\alpha_{i}(t) \leq 0 \quad \alpha_{i}(t):=\max _{s \in(0, t)} \varepsilon_{i}(s),
$$

with the damage functions $\phi_{i}$. For $\phi_{i}<0$ no evolution of damage occurs, and at $\phi_{i}=0, \alpha_{i}$ evolves only in the case of loading $\varepsilon_{i}>0$. For the damage variable $d_{i}$, we take a simple discontinuous evolution law

$$
d_{i}= \begin{cases}\bar{d} & \text { if } \alpha_{i}(t)-\varepsilon_{2} \geq 0 \\ 0 & \text { otherwise }\end{cases}
$$



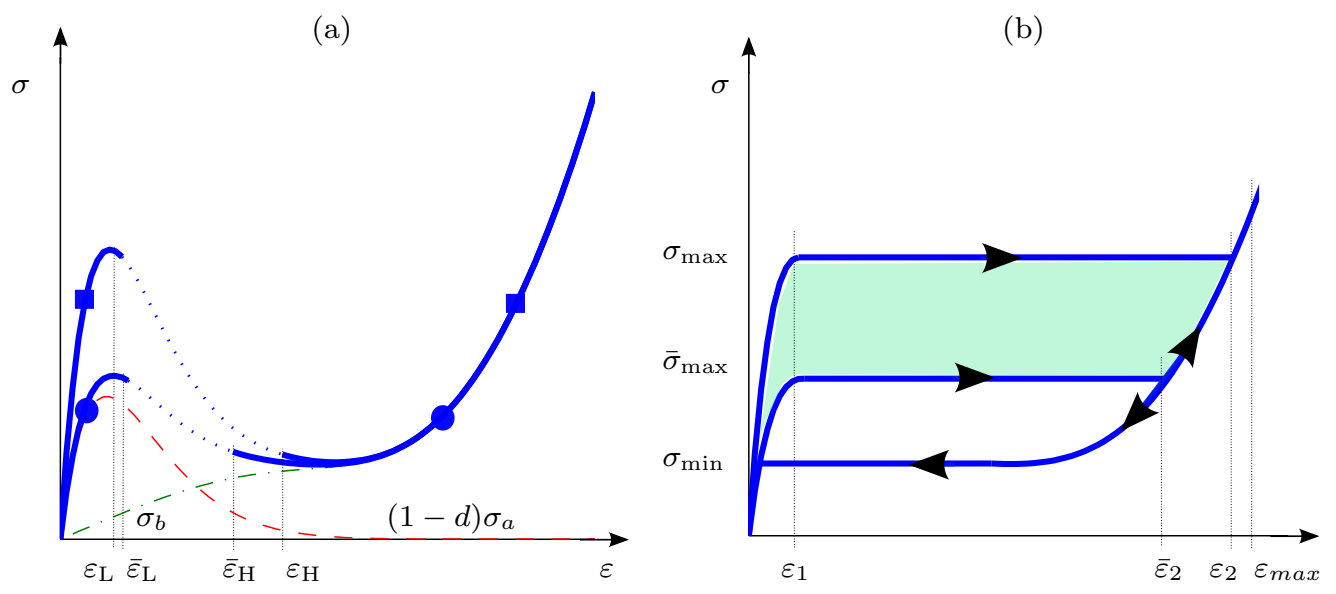

Fig. 4 (a) Graph $\left(w^{\prime}, \varepsilon_{i}\right)$ of a undamaged (square marks) and a damaged (circle marks) spring. (b) Response curves of the model to two loading-unloading cycles with maximal deformation $\varepsilon_{\max }$, when the rate-dependent effects are neglected.

where $0<\bar{d}<1$ and $\varepsilon_{2}$ is the strain at which the thermodynamic force $f_{i}$, that is identical to $w_{a}$, reaches its maximum value, see Figure $2 \mathrm{a}$. Note in Figure $2 \mathrm{~b}$ that $\varepsilon_{2}$ corresponds to the local minimum of the response curve of the spring. The evolution law (11) expresses the idea that the damage occurs when the deformation of the spring reaches the second ascending branch for the first time. In Section 5 , the value of $\bar{d}$ will be identified from the experimental cyclic response curves.

In order to illustrate and clarify the effects of damage on the springs and on the response curves, we give:

- on Figure 4a the graph $(\sigma, \varepsilon)$ for a damaged and an undamaged spring.

- on Figure 4b the response curves of the chain to two loading-unloading cycles with maximal deformation $\varepsilon_{\max }$, when rate dependence is neglected. The area in gray shows the dissipation energy due to damage.

Del Piero and Pampolini [3] showed that for the chain in Figure 3 subjected to the deformation process $t \rightarrow \varepsilon_{0}(t)$, stable ${ }^{4}$ solutions are those in which each spring deformation $\varepsilon_{i}$ satisfies the condition

$$
\frac{\partial^{2} w\left(\varepsilon_{i}, d_{i}\right)}{\partial \varepsilon_{i}^{2}}+\sum_{j=1}^{J} k_{j}>0
$$

for all $t$. Denote with $\left(\varepsilon_{\mathrm{L}}, \varepsilon_{\mathrm{H}}\right)$ and with $\left(\bar{\varepsilon}_{\mathrm{L}}, \bar{\varepsilon}_{\mathrm{H}}\right)$ the deformation values that satisfy (12) as an equality with $d_{i}=0$ and $d_{i}=\bar{d}$, respectively ${ }^{5}$. The stability condition (12) is therefore satisfied for $\varepsilon_{i}$ in the intervals $\left\{0 \leq \varepsilon_{i}<\varepsilon_{\mathrm{L}}, \varepsilon_{i}>\varepsilon_{\mathrm{H}}\right\}$ and $\left\{0 \leq \varepsilon_{i}<\bar{\varepsilon}_{\mathrm{L}}, \varepsilon_{i}>\bar{\varepsilon}_{\mathrm{H}}\right\}$ in the case of virgin and damaged springs, respectively. Since $\bar{\varepsilon}_{\mathrm{L}}>\varepsilon_{\mathrm{L}}$ and $\bar{\varepsilon}_{\mathrm{H}}<\varepsilon_{\mathrm{H}}$, the damage law here introduced has the effect of increasing the amplitude of the interval of $\varepsilon_{i}$ that satisfy the stability condition (12), see Figure 4a. As it will be shown in Section 6 this effect plays a crucial role in the evolution of the deformation during a cyclic process.

In Figure $4 \mathrm{~b}$ we show the response of the model to two loading-unloading cycles with maximal deformation $\varepsilon_{\max }$, when the deformation is applied sufficiently slowly so that the rate-dependent effects can be neglected. In this case, the response curves are constructed as in the purely elastic case presented in [24]. Starting from the initial homogeneous configuration $\varepsilon_{i}=0, \forall i$, the system follows the first ascending branch till the force reaches the value $\sigma_{\max }$. At this point, the homogeneous configuration becomes unstable and the springs progressively pass from the low-deformation phase $\left(\varepsilon_{i}=\varepsilon_{1}\right)$ to the high deformation phase $\left(\varepsilon_{i}=\varepsilon_{2}\right)$. When the total imposed deformation $\varepsilon_{0}$ overcomes the value $\varepsilon_{2}$ all the springs have changed phase and for further increase of the deformation the system

\footnotetext{
4 Stability with respect initial data perturbation is considered.

5 Here we assume that the material constants are taken so that there are always two distinguished deformation values that satisfy condition (12) as an equality.
} 
follows the second ascending branch. At unloading one observes the same behavior but the change of phase starts when the force reaches the value $\sigma_{\min }$. In the second cycle since the springs are damaged, the response curve at loading differs from that of the first cycle. In particular:

- the slope of the first ascending branch decreases;

- the changes of phase occur at a lower value of force, $\bar{\sigma}_{\max }$;

- in the plateau we still have the progressive change of phase of the springs but the springs in high-deformation phase have deformation $\bar{\varepsilon}_{2}$.

At unloading, the response curve does not change in the second cycle, and therefore a reduction of the amplitude of the hysteresis loop is observed.

In the case of interest here where both viscous effects and damage are taken into account, the response of the model can be computed via numerical simulations by using the approximation presented in the following Section.

\section{Numerical implementation}

\subsection{Numerical approximation}

For a given deformation process $t \rightarrow \varepsilon_{0}(t)$, the evolution problem consists in finding the force $\sigma(t)$ and for each element the deformation $\varepsilon_{i}(t)$, which satisfy equations (1), (4), (5), (7) and (9)a for all $t$, under given initial conditions $\varepsilon_{i}(0)=0, \sigma_{i j}^{d}(0)=0, \sigma_{h}^{v}(0)=0$.

By differentiation of $(7)$, the relation

$$
\dot{\sigma}(t)=\dot{\sigma}_{i}^{e}(t)+\dot{\sigma}_{i}^{d}(t)+\dot{\sigma}^{v}(t),
$$

follows, and by using relations (4), (5) and (9)a, we obtain

$$
\dot{\sigma}(t)=k_{e}\left(\varepsilon_{i}(t)\right) \dot{\varepsilon}_{i}(t)+\sum_{j=1}^{J}\left(k_{j} \dot{\varepsilon}_{i}(t)-p_{j} \sigma_{i j}^{d}(t)\right)+\sum_{h=1}^{H}\left(k_{h} \dot{\varepsilon}_{0}(t)-p_{h} \sigma_{h}^{v}(t)\right),
$$

where

$$
\begin{aligned}
k_{e}\left(\varepsilon_{i}\right)=\mu-\beta \exp \left(-k(a-1)^{2}\right)+\mu(1 & \left.+\varepsilon_{i}\right)^{-2} \\
-2 k \beta(1+ & \left.\varepsilon_{i}-a\right) \exp \left(-k\left(1+\varepsilon_{i}-a\right)^{2}\right) \\
& +c\left(1-d_{i}\right)\left(1+\varepsilon_{i}\right)^{m}\left(m+1-(m-1)\left(1+\varepsilon_{i}\right)^{-2}\right) .
\end{aligned}
$$

An approximate numerical solution is obtained with a classical time discretization $t_{n}=n \Delta t$ (where $\Delta t$ is the time step) and assuming the strain rates $\dot{\varepsilon}_{i}$ to be constant in each interval. Once the solution has been determined up to time $t^{n}$, the rates $\left(\dot{\sigma}\left(t^{n}\right), \dot{\varepsilon}_{i}\left(t^{n}\right)\right)$ can be computed by solving the linear system

$$
\begin{aligned}
& \dot{\sigma}\left(t^{n}\right)=Q_{i} \dot{\varepsilon}_{i}\left(t^{n}\right)+A_{i}^{n}, \quad i=1,2, \ldots N \\
& \sum_{i=1}^{N} \dot{\varepsilon}_{i}\left(t^{n}\right)=N \dot{\varepsilon}_{0}
\end{aligned}
$$

where

$$
\begin{aligned}
& Q_{i}=k_{e}\left(\varepsilon_{i}\left(t^{n}\right)\right)+\sum_{j=1}^{J} k_{j}, \\
& A_{i}=\sum_{h=1}^{H}\left(k_{h} \dot{\varepsilon}_{0}-p_{h} \sigma_{h}^{v}\left(t^{n}\right)\right)-\sum_{j=1}^{J} p_{j} \sigma_{i j}^{d}\left(t^{n}\right) .
\end{aligned}
$$

The system (16) has a unique solution if the matrix $R$ 


$$
R=\left[\begin{array}{ccccc}
Q_{1} & 0 & 0 & \ldots & -1 \\
0 & Q_{2} & 0 & \ldots & -1 \\
0 & 0 & Q_{3} & \ldots & -1 \\
\ldots & \ldots & \ldots & \ldots & \ldots \\
1 & 1 & 1 & \ldots & 0
\end{array}\right]
$$

is not singular. If we consider an initial homogeneous configuration where all $A_{i}$ and $Q_{i}$ are the same for all $i$, there exists a time interval $(0, \bar{t})$ in which the unique solution of the system (16) is the homogeneous solution

$$
\dot{\varepsilon}_{i}=\dot{\varepsilon}_{0} \quad \dot{\sigma}=Q_{i} \dot{\varepsilon}_{0}+A_{i} .
$$

This solution becomes unstable at the time $\bar{t}$ at which all coefficients $Q_{i}$ become zero. In this case, a stable solution, in which the deformation localizes at a single spring, can be obtained by assuming a slight dispersion of the data (see [3] for details).

To compute this localized solution numerically the differential equations (5) are discretized using an explicit Euler scheme. Preliminary tests with various methods (Runge-Kutta, Heunn) showed that the simple Euler method was convenient. Then the following non linear system is obtained and solved in terms of the unknowns $\left(\sigma\left(t^{n+1}\right), \varepsilon_{i}\left(t^{n+1}\right)\right)$,

$$
\begin{aligned}
& \sigma\left(t^{n+1}\right)=\sigma\left(t^{n}\right)+\sigma_{i}^{e}\left(\varepsilon_{i}\left(t^{n+1}\right)\right) \\
&+\sum_{j=1}^{J}\left(k_{j}\left(\varepsilon_{i}\left(t^{n+1}\right)-\varepsilon_{i}\left(t^{n}\right)\right)+\left(1-p_{j} \Delta t\right) \sigma_{i j}^{d}\left(t^{n}\right)\right) \\
&+\sum_{h=1}^{H}\left(k_{h}\left(\varepsilon_{0}\left(t^{n+1}\right)-\varepsilon_{0}\left(t^{n}\right)\right)+\left(1-p_{h} \Delta t\right) \sigma_{h}^{v}\left(t^{n}\right)\right), \\
& \sum_{i=1}^{N} \varepsilon_{i}\left(t^{n+1}\right)=N \varepsilon_{0}\left(t^{n+1}\right) .
\end{aligned}
$$

The numerical procedure used can be summarized as follows. At each time step, compute the determinant of the matrix of coefficients in the system (16); if the determinant has not changed sign since the previous time step, solve the linear system (16). Otherwise, a change of phase is assumed to have occurred in one spring, and the solution is obtained by solving the non linear system (19) with a Newton-Raphson algorithm.

\subsection{Number of elements and time steps}

The numerical scheme presented in the previous Section was implemented in a Matlab environment, and loading-unloading cycles with different time steps $\Delta t$ and different elements number $N$ were computed. The results are shown in Figure 5. Table 1 gives some details about the computational times.

As in the purely elastic model [24] by increasing the number of elements one obtains smoother response curves, i.e. the amplitudes of the stress jumps occurring during the phase transitions decrease as shown in Figure 5a. These oscillations become negligible when a chain of 120 elements is used.

In the cyclic test simulations, the time step $\Delta t=0.03 \mathrm{~s}$ was adopted. Taking a larger time step would generate significant errors in the discrete approximation of the solution at the end of the first cycle (see Figure $5 \mathrm{~b}$ ). In the simulation of the resting periods with duration up to two days, two time steps were used: $\Delta t=0.03 \mathrm{~s}$ during the first $30 \mathrm{~min}$ and $\Delta t=10 \mathrm{~s}$ thereafter. Small time steps are necessary during the first few minutes to account for the fast relaxation effects of the foam. A second, larger time step makes it possible to reduce the computational time. Details about the computational time involved in various tests are presented in Table 2 . The numerical method proposed here was also used in [3]. 
(a)
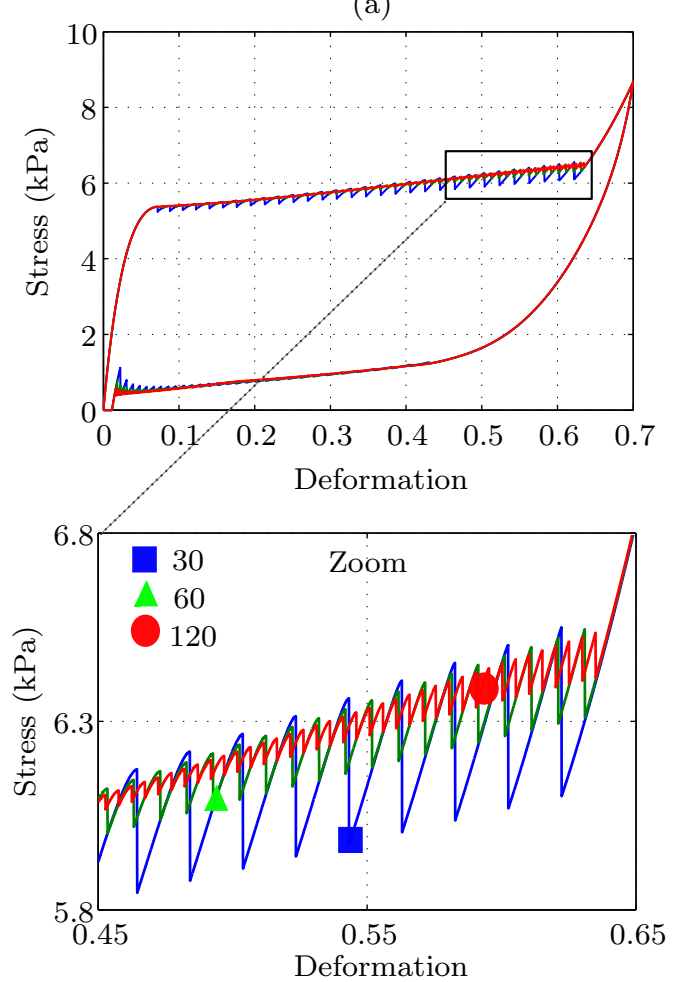

(b)
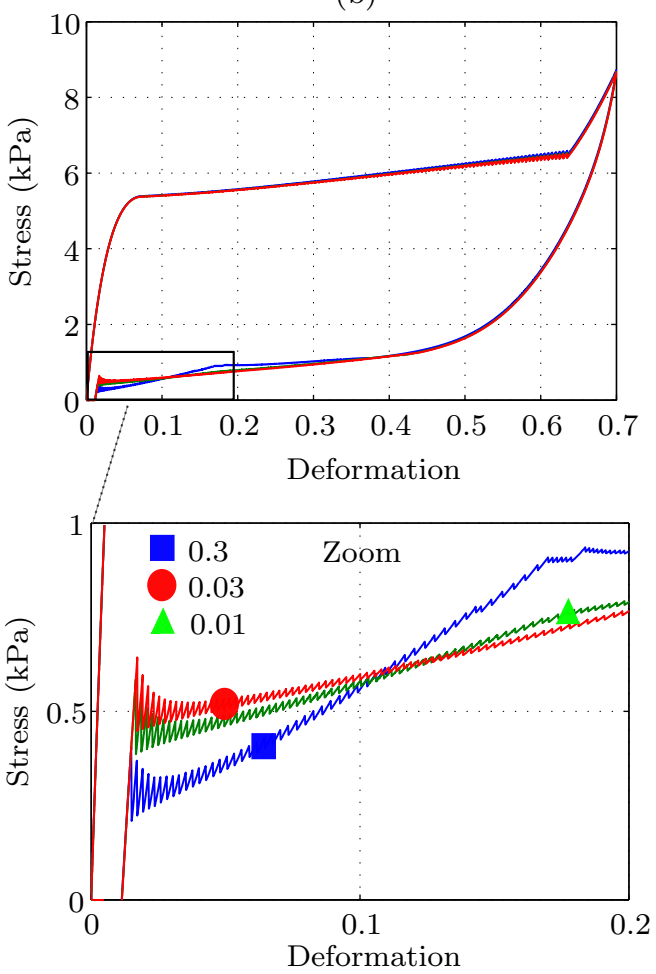

Fig. 5 Simulation of a loading-unloading cycle with various numbers of elements $N$ in (a) : 30 (square marks), 60 (triangle) and 120 (circle) for $\Delta t=0.03 \mathrm{~s}$; and with various time steps $\Delta t$ in (b) : $0.3 \mathrm{~s}$ (square), $0.03 \mathrm{~s}$ (circle) et $0.01 \mathrm{~s}$ (triangle), for $N=120$.

Table 1 Influence of the number of elements $N$ and of the time step $\Delta t$ on the computational time in the case of a loading-unloading cycle at $5 \mathrm{~mm} / \mathrm{min}$ with a maximum deformation of 0.7 (duration of the experiment: $14 \mathrm{~min})$.

\begin{tabular}{lcr}
\hline$N$ & $\Delta t$ & Computational time \\
\hline 30 & $0.03 \mathrm{~s}$ & $1 \mathrm{~min} 15 \mathrm{~s}$ \\
60 & $0.03 \mathrm{~s}$ & $1 \mathrm{~min} 40 \mathrm{~s}$ \\
120 & $0.03 \mathrm{~s}$ & $2 \mathrm{~min} 38 \mathrm{~s}$ \\
120 & $0.01 \mathrm{~s}$ & $8 \mathrm{~min}$ \\
120 & $0.3 \mathrm{~s}$ & $22 \mathrm{~s}$. \\
\hline
\end{tabular}

Table 2 Computational time involved in various simulations $(N=120)$.

\begin{tabular}{lcr}
\hline Test & Duration of the experiment & Computational time \\
\hline One cycle & $14 \mathrm{~min}$ & $2 \mathrm{~min} 38 \mathrm{~s}$ \\
Four cycles & $56 \mathrm{~min}$ & $11 \mathrm{~min}$ \\
Resting periods & 2 days & $24 \mathrm{~min}$ \\
\hline
\end{tabular}

\section{Identification of the material constants}

\subsection{Identification of the viscosity constants}

Relaxation tests were performed in order to identify the parameters $k_{j}, p_{j}, k_{h}, p_{h}$ of the dissipative elements. In the identification procedure, we take into account the initial step of the relaxation experiments, i.e. the loading ramp used to reach the prescribed deformation value at which relaxation starts $^{6}$. The results of a 10 -day relaxation test at $\varepsilon_{0}=0.7$ are shown in Figure 6 . Note that a rapid decrease in the force occurs during the first few minutes, followed by a long relaxation, which is still not complete 10 days later.

\footnotetext{
6 We carried out relaxation tests with the same loading ramp velocity used in the cyclic tests $(5 \mathrm{~mm} / \mathrm{min})$
} 


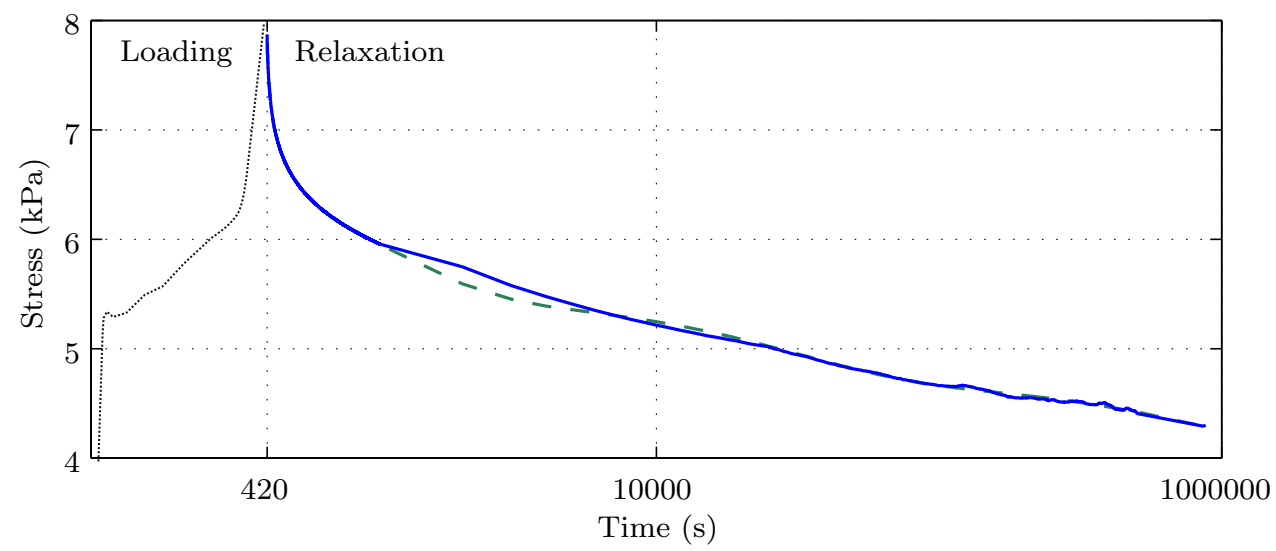

Fig. 6 Comparison between the experimental relaxation curves (full lines) and the model ones (dashed lines) with the parameters given in Table 4 . Prescribed deformation $\varepsilon_{0}=0.7$ obtained by applying a loading ramp at a speed of $5 \mathrm{~mm} / \mathrm{min}$.

There are two series of Maxwell elements in the model: those located inside the chain, and those in the single dissipative element located outside the chain. For the prescribed deformation $\varepsilon_{0}=0.7$, the cells are totally compressed, the deformation is almost homogeneous and the relaxation effect is the sum of the contributions of the two series of Maxwell elements. The total force $\sigma$ in a relaxation process with the maximum deformation $\bar{\varepsilon}$ and the crosshead speed $\dot{\bar{\varepsilon}}$ can be written as follows:

$$
\sigma(t)=\sigma_{i}^{e}(\bar{\varepsilon})+\sum_{j=1}^{J+H} K_{j} \exp \left(-p_{j} t\right), \quad \text { for } t>\bar{\varepsilon} / \dot{\bar{\varepsilon}},
$$

with

$$
K_{j}=\frac{k_{j}}{p_{j}} \dot{\bar{\varepsilon}}\left(\exp \left(p_{j} \frac{\dot{\bar{\varepsilon}}}{\bar{\varepsilon}}\right)-1\right)
$$

and $k_{j}, p_{j}$ are the material constants of the internal Maxwell elements for $j \leq J$, and those of the external Maxwell elements for $j>J$.

In order to identify the parameters of the viscoelastic elements, the experimental curve was approximated with a sum of exponentials. The inelastic parameters $\left(K_{j}, p_{j}\right)$ were obtained by minimizing the functional

$$
F\left(K_{j}, p_{j}\right)=\sum_{i=1}^{L}\left(y\left(t_{i}\right)-\sum_{j=1}^{J} K_{j} \exp \left(-p_{j} t_{i}\right)\right)^{2},
$$

where $y\left(t_{i}\right)=\tilde{y}\left(t_{i}\right)-\sigma_{i}^{e}(\bar{\varepsilon})$, and $\left(t_{i}, \tilde{y}\left(t_{i}\right)\right)$ are $L$ points taken on the experimental relaxation curve. $J$ is the minimum number of exponentials required to obtain a satisfactory picture of the relaxation curve. $J$ is usually quite small (two or three), but in the present case, the description of relaxation effects including short and large time scales was found to require at least five exponentials.

Before using optimization algorithms, we made a preliminary estimate of the parameters. For this purpose, two methods were considered: a geometrical method [13] based on a logarithmic graphic analysis of the relaxation curve, and the Prony method [26].

In the first case, the curve $(t, \ln y)$ is plotted, and its final part is fitted with a straight line. The slope and the value at the origin determine $p_{1}$ and $K_{1}$, respectively. The first exponential can thus be identified and the curve $(t, \ln \bar{y})$, with $\bar{y}=y-K_{1} \exp \left(-p_{1} t\right)$, can be plotted. Repeating the procedure on this curve gives an estimate of the coefficients of the second exponential. The coefficients of the other exponentials are determined in the same way.

The Prony method is more precise. The $2 J$ coefficients $\left(K_{j}, p_{j}\right)$ are obtained by making the sum of exponentials to cross through $2 J$ equidistant time points on the experimental curve. This gives the system of $2 J$ non linear equations 
Table 3 Convergence of the Hooke and Jeeves method (3320 points on the experimental curve).

\begin{tabular}{lccr}
\hline$J$ & Iter. & $F$ & Computational time \\
\hline 3 & 578 & $6.1 \times 10^{-3}$ & $2 \mathrm{~min}$ \\
4 & 2458 & $4.4 \times 10^{-4}$ & $10 \mathrm{~min}$ \\
5 & 2001 & $3.3 \times 10^{-5}$ & $11 \mathrm{~min}$ \\
\hline
\end{tabular}

Table 4 The material constants of the Maxwell elements identified on the relaxation curve at $5 \mathrm{~mm} / \mathrm{min}$

\begin{tabular}{lccc}
\hline$j$ & $k_{j}[\mathrm{kPa}]$ & $p_{j}\left[\mathrm{~s}^{-1}\right]$ & $h_{j}$ \\
\hline 1 & 0.99 & $9.64 \times 10^{-7}$ & 0 \\
2 & 1.09 & $3.13 \times 10^{-5}$ & 1 \\
3 & 1.81 & $1.04 \times 10^{-3}$ & 0 \\
4 & 5.25 & $1.20 \times 10^{-2}$ & 1 \\
5 & 34.8 & $9.41 \times 10^{-2}$ & 0 \\
\hline
\end{tabular}

$$
\begin{aligned}
& K_{1}+K_{2}+\cdots+K_{J}=y(0) \\
& K_{1} u_{1}+K_{2} u_{2}+\cdots+K_{J} u_{J}=y(\lambda) \\
& K_{1} u_{1}^{2}+K_{2} u_{2}^{2}+\cdots+K_{J} u_{J}^{2}=y(2 \lambda) \\
& \vdots \\
& K_{1} u_{1}^{J-1}+K_{2} u_{2}^{2 J-1}+\cdots+K_{J} u_{J}^{2 J-1}=y((2 J-1) \lambda),
\end{aligned}
$$

where $u_{j}=\exp \left(-p_{j} \lambda\right)$ and $\lambda$ is the time step. The idea here is to transform the system (22) to two systems of $J$ linear equations. This can be done by taking the sum of exponentials to be the solution of the homogeneous difference equation

$$
\begin{aligned}
y((J+L) \lambda)+C_{1} y((J+L-1) \lambda)+C_{2} y((J+L-2) \lambda) & +\ldots \\
& +C_{L} y(L)=0, \quad L=1,2, \ldots, J .
\end{aligned}
$$

This is a linear system in terms of the unknowns $C_{L}$. Then $-p_{j}$ are the roots of the characteristic polynomial

$$
p^{L}-C_{1} p^{L-1}-C_{2} p^{L-2}-\cdots-C_{L}=0 .
$$

Once $p_{j}$ have been determined, the system (22) reduces to a system of $J$ linear equations in terms of the unknowns $K_{j}$. Details of this procedure are given in [26].

For the optimization algorithm, a gradient method with optimum parameter (with parabolic estimate) was tested, but an accurate determination of the optimum parameter turned out to be difficult to achieve and this method was therefore excluded. On the contrary, the simple and robust Hooke and Jeeves method [14] turned out to be highly efficient and gave satisfactory results in a quite short computational time (see Table 3). Consequently the identification of the viscous parameters, i.e. the minimization of (21), is conducted by using the Hookes and Jeaves method with a good initial condition obtained with the Prony method. This procedure was applied here to the relaxation experiment and it was shown that five exponentials, with the parameter values given in Table 4 , reproduce the relaxation curve satisfactorily at both small and large times, as shown in Figure 6 .

The relaxation test makes it possible to characterize the five Maxwell elements, but it does not provide any information about their distribution between the internal and external dissipative elements. This information is obtained by the analysis of the response under uniaxial compression. Five parameters $h_{j}$, which determine the contribution of each Maxwell element to the internal and external dissipative elements, were introduced. It was assumed that $h_{j}$ can take only two values: " 1 " if the Maxwell element belongs to the internal dissipative element and " 0 " if it belongs to the external one. Different distributions of the five Maxwell elements give response curves with different slopes in the plateau regime, see Figure 7. A good picture of the slope of the response curve in the plateau regime was obtained with the values of $h_{j}$ given in Table 4 . These values correspond to taking two Maxwell elements in the internal dissipative element and three in the external one. 


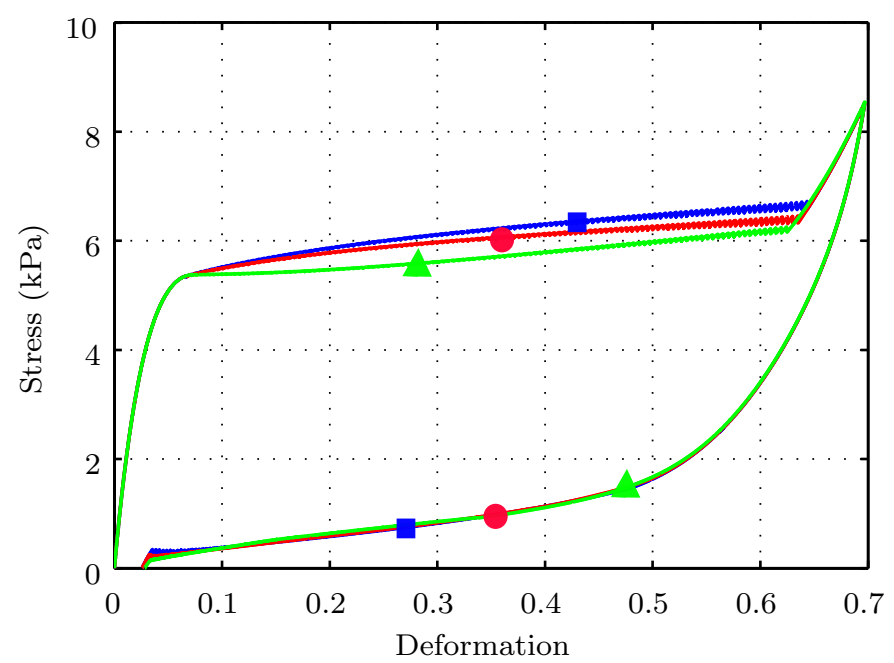

Fig. 7 Simulation results of a loading-unloading cycle for different distribution of the Maxwell elements among the internal $\left(h_{j}=0\right)$ and external $\left(h_{j}=1\right)$ dissipative elements: square marks for $h_{j}=(1,1,0,0,0)$; circles for $h_{j}=(1,0,1,0,0)$ and triangles for $h_{j}=(0,0,1,1,0)$. The sequence of $j-$ follows that used in Table 4 .

\subsection{Identification of the elastic constants}

The constants $(c, \mu, m, \beta, k, a)$ of the non linear elastic springs are identified on loading-unloading cyclic experiments, taking the constants of the Maxwell elements to be those identified in the relaxation experiments and given in Table 4.

Before starting the identification procedure, we analyzed the role of each constant on the response curve $\left(\sigma^{e}, \varepsilon\right)$ of the non linear spring. It can be seen from Figure 8 that:

- $c$ determines the initial slope and the value of the local maximum, Figure 8a;

$-\mu$ determines the second ascending branch, Figure 8b;

- $m$ determines the descending branch, the position and the value of the local maximum, Figure 8c;

- when $\beta$ increases, $\sigma^{e}$ decreases when $\varepsilon \simeq a$, Figure $8 \mathrm{~d}$;

$-k$ determines the slope of the curve when $\varepsilon \simeq a$, Figure 8e;

- $a$ determines essentially the position of the local minimum, Figure $8 \mathrm{f}$.

Note in (4) that the last term of $\sigma_{b}$ is a Gaussian function characterized by the constants $a, \beta$ and $k ; a$ and $\beta$ determine the position and the value of the peak, respectively, and $k$ determines the standard deviation.

After this preliminary analysis, the identification procedure was performed on numerical simulations of a complete loading-unloading cycle using the model with 120 elements and a time step of $0.03 \mathrm{~s}$ (see Section 4.2). The values of the constants of the viscoelastic elements are those given in Table 4. The values of the elastic constants were identified on the average response curve of three loading-unloading tests obtained with a crosshead speed of $5 \mathrm{~mm} / \mathrm{min}$. The following procedure was used for this purpose:

1. select $c$ to obtain the initial slope of the experimental curve;

2. select $m$ (with $c$ fixed) to obtain the appropriate value of the force at the beginning of the plateau regime;

3. select $\mu$ (with $c$ and $m$ fixed) to obtain the appropriate value of the force at the end of the loading process $(\varepsilon=0.7)$;

4. perform an optimization routine with the Hooke and Jeeves method to determine the values of $a$ $\beta$ and $k$.

The results obtained with this identification procedure are shown in Figure 9a, and the values of the elastic constants are given in Table 5. 

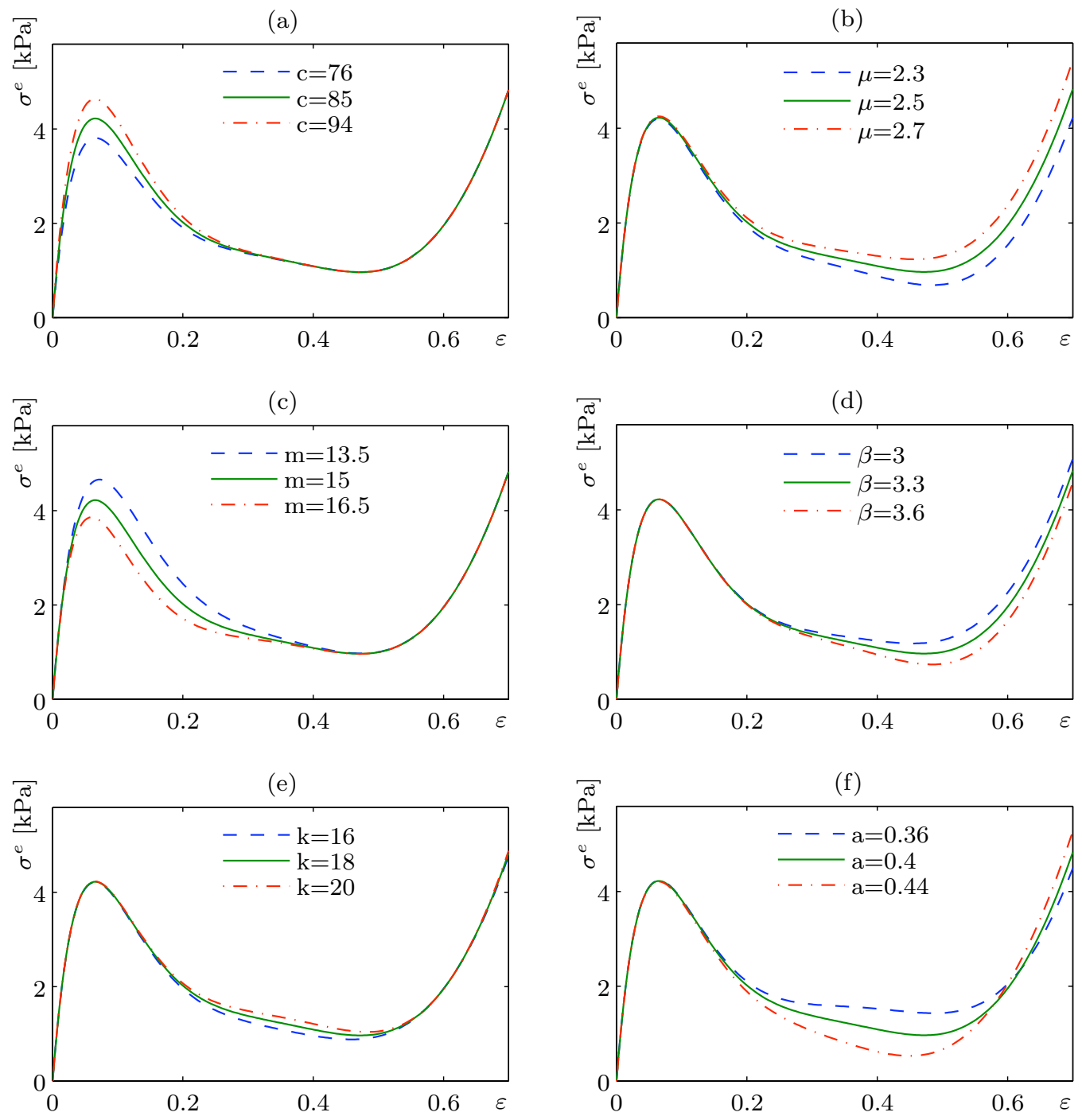

Fig. 8 Influence of the elastic parameters on the graph $\left(\sigma^{e}, \varepsilon\right)$.

(a)

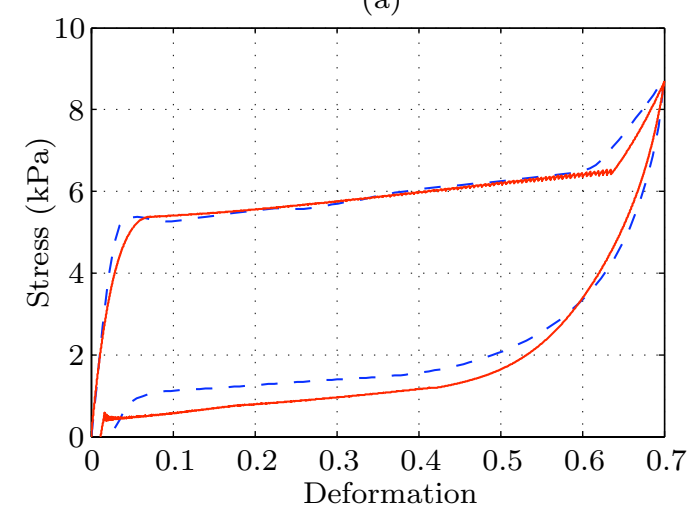

(b)

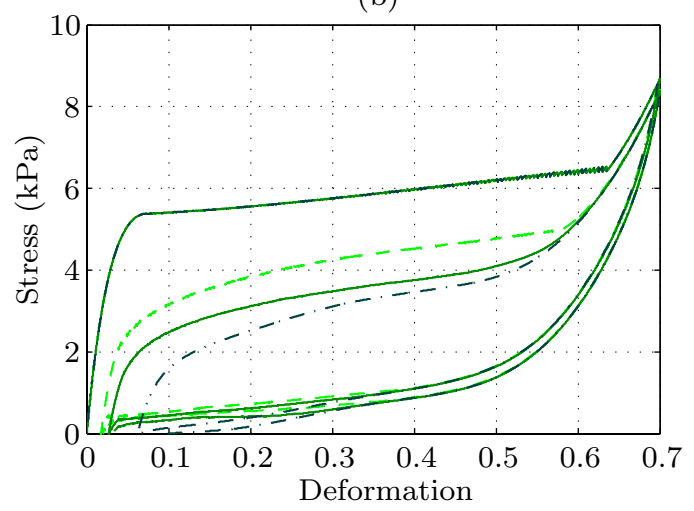

Fig. 9 (a) Result after the identification of the elastic constants (dashed line: experiments; full line: model). (b) First and second cycle response curves with three different values of the damage parameter $\bar{d}$ : 0.7 (dashed line), 0.5 (full line), and 0.3 (dashed-dotted line). 
Table 5 The values of the elastic constants

\begin{tabular}{llllll}
\hline$c=85 \mathrm{kPa}$ & $m=15$ & $\mu=2.5 \mathrm{kPa}$ & $a=0.4$ & $\beta=3.3 \mathrm{kPa}$ & $k=18$
\end{tabular}

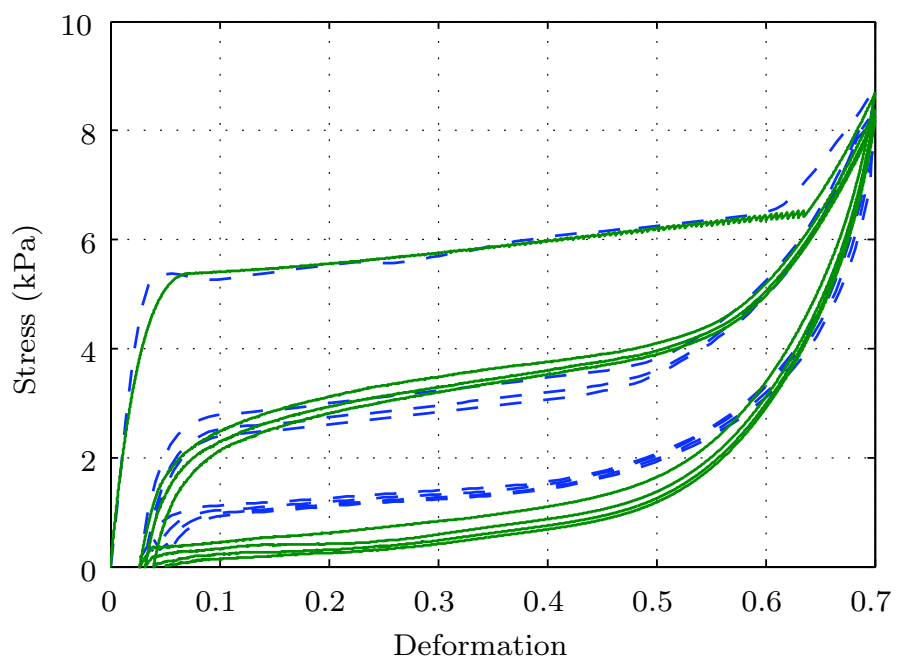

Fig. 10 Numerical simulations (full lines) and experiments (dotted lines) for four cycles of loading and unloading.

\subsection{Identification of the damage parameter}

The value of $\bar{d}$ was identified from the experimental cyclic response curves obtained at a speed of 5 $\mathrm{mm} / \mathrm{min}$. In Figure $9 \mathrm{~b}$, we show the results of the simulation of two loading-unloading cycles with three different values of $d$. A satisfactory picture of the cyclic behavior was obtained with $\bar{d}=0.5$, see Figure 10. The introduction of damage not only determines the decrease of the force in the plateau, but also reduces the length of the plateau in agreement with the experimental data. However, one sees that in the simulations the value of the stress in the plateau at unloading is slightly underestimated.

\section{Numerical simulations}

\subsection{Progressive mixing of the two phases}

A picture of the localization phenomenon is given in Figure 11, where the deformation of the 120 elements in the chain is shown at eight different steps in the response curve during a cyclic compression simulation.

In the first almost linear part of the curve the deformation of the chain is homogeneous (see step A). From step A to step D, in the plateau, the localization of the deformation can be clearly observed. At B and C, two separate zones corresponding to the two phases of the foam are characterized by deformation values of around 0.08 and 0.6 , respectively. When all elements have changed phase, they have almost the same value of deformation (step D). At unloading for $\varepsilon_{0}=0.45$, the deformation of each element of the chain stays close to $\varepsilon_{0}$ with values ranging between 0.35 and 0.5 (step E). When the prescribed deformation decreases, a phase transition occurs, and at $\varepsilon_{0}=0.12$ (step F), the elements are again divided into two groups, corresponding to the two phases of the material. At the beginning of the second cycle (step G), all the elements are in the low deformation phase with slightly different values of deformation. In the plateau, the behavior is very different from what observed during the first cycle. The heterogeneity of the deformation does not take the form of two separate zones, but that of a single diffuse non homogeneous zone (step $\mathrm{H}$ ). These differences are experimentally observed, see Figure 12. 

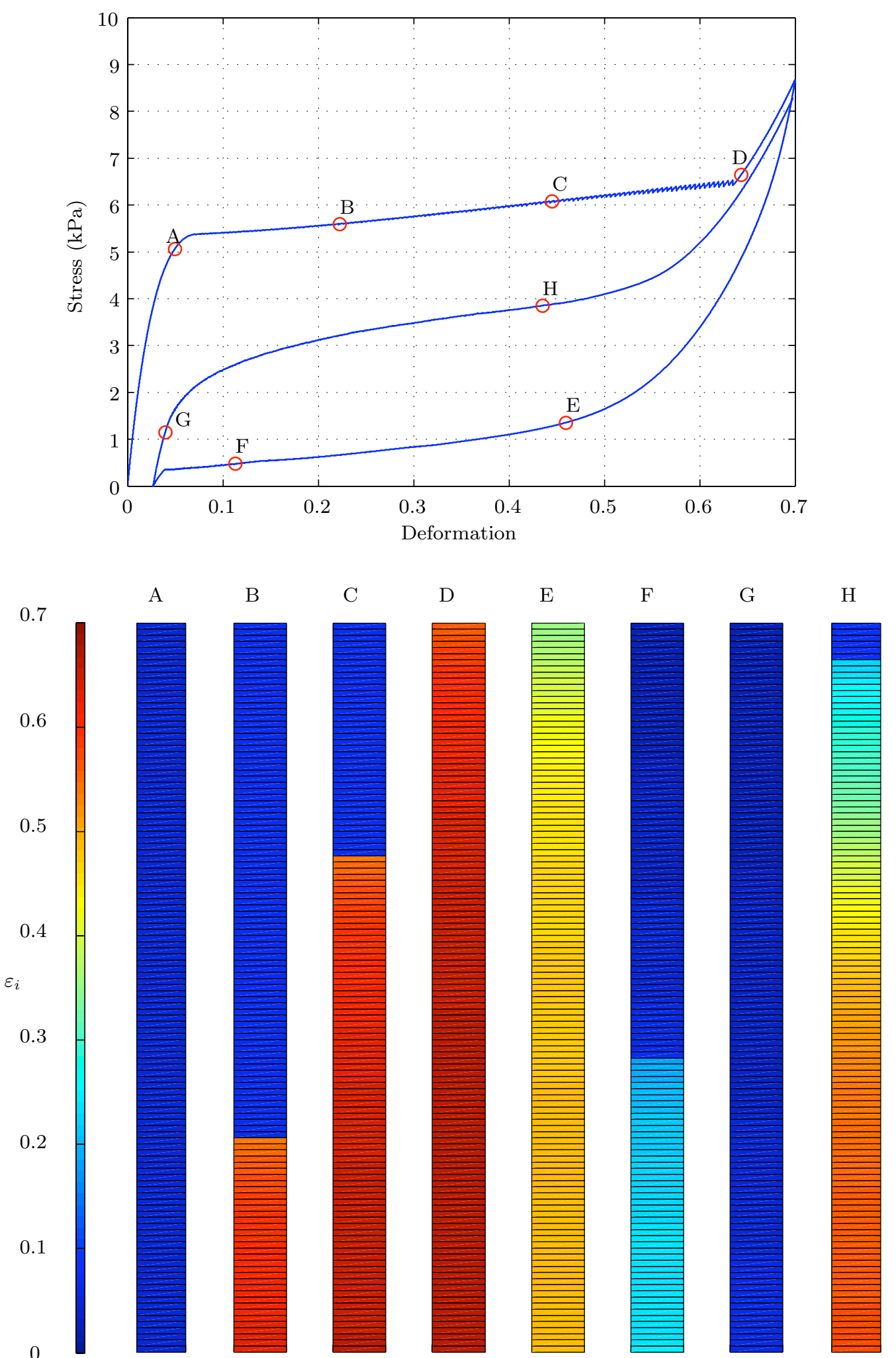

Fig. 11 Evolution of the deformation in the chain of 120 elements during the simulation. A, D, E and G are single phase configurations, while $\mathrm{B}, \mathrm{C}, \mathrm{F}$ and $\mathrm{H}$ are two phase configurations. Note in $\mathrm{B}, \mathrm{C}$ and $\mathrm{F}$ the two separate blocks. 

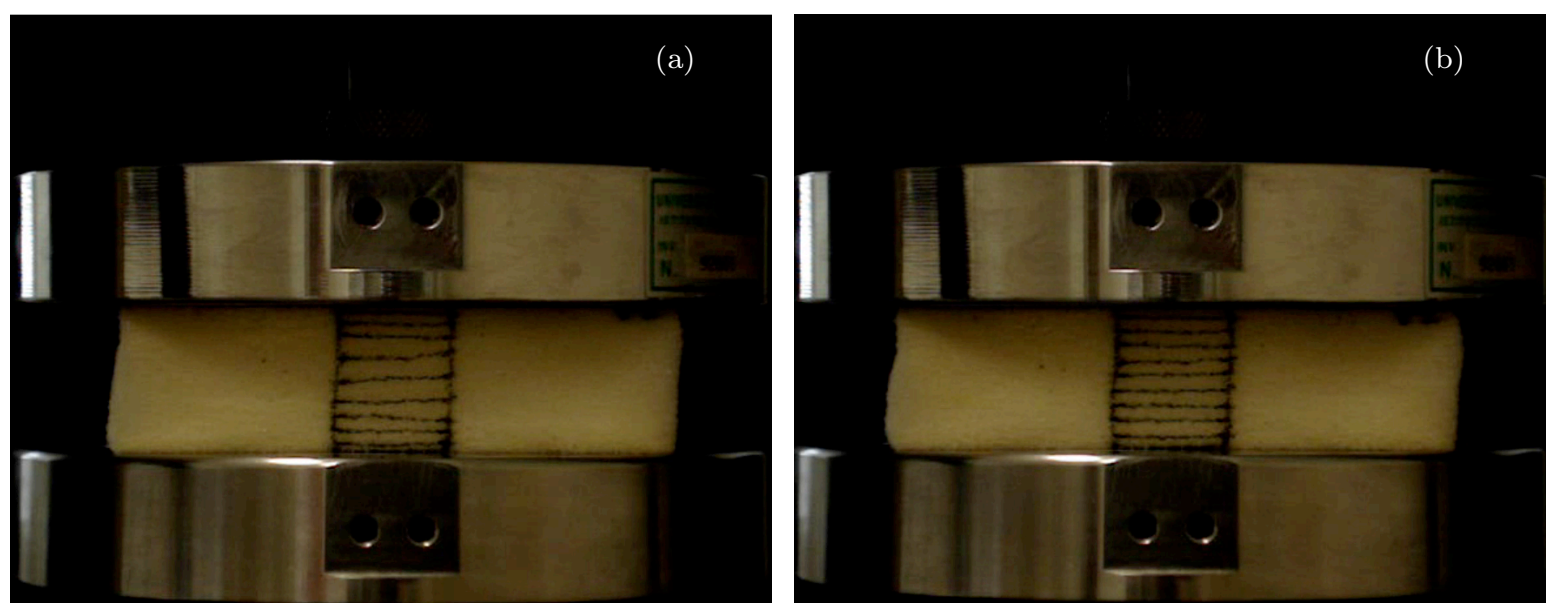

Fig. 12 Deformation of a open-cell polyurethane foam in the first (a) and second (b) loading cycle for the same value of the total prescribed deformation $\varepsilon_{0}=0.45$, corresponding to step $\mathrm{C}$ and step $\mathrm{H}$ in Figure 11 , respectively. In (a) we can distinguish an high-deformation region at the plate ends and a low-deformation region in the center. In (b) the two regions are not clearly detectable.

(a)

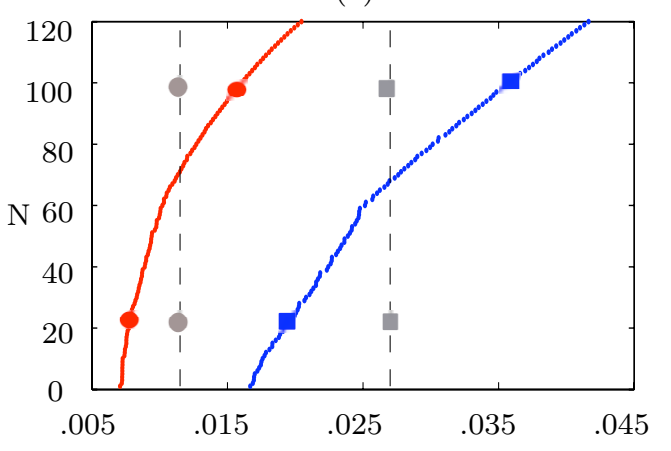

(b)

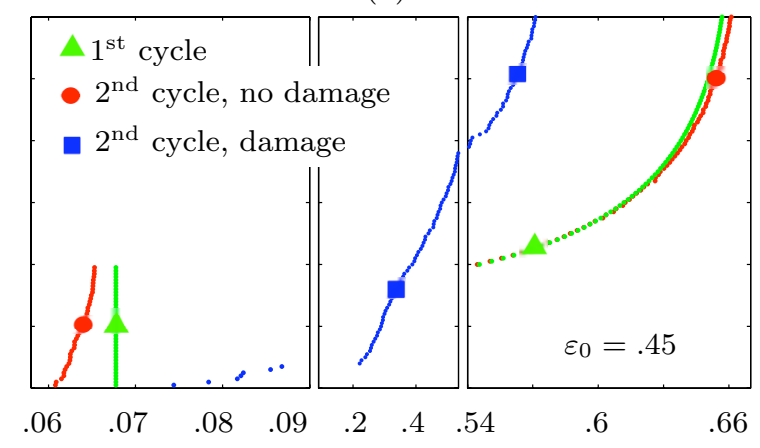

Deformation

Fig. 13 Simulation results of the deformation of the 120 elements at the end of the first cycle (a), and at loading for $\varepsilon_{0}=0.45$ in the first and in the second cycle (b). Square and circular marks distinguish the solutions with or without damage, respectively. Dashed lines in (a) show the total residual deformation after the first cycle.

Similarly to the elastic case [24], in the first cycle the initial configuration is homogeneous, and so all elements reach the instability condition $Q_{i}=0$ at the same time. At this point one element changes phase and the others stay grouped at a lower value of deformation. Two blocks of elements are therefore created. When the prescribed deformation increases, other changes of phase occur and the number of elements in the high deformed block increases.

In the subsequent cycles, a different scenario is observed because of damage. To illustrate this point we show in Figure 13 the deformation of the 120 elements at different stages of the loading process for the two cases with and without damage.

As shown in Figure 13a, the residual deformation at the end of the first loading-unloading cycle (point $\mathrm{G}$ in Figure 11) is not homogeneously distributed on all the elements: elements have a different residual deformation because of their different deformation history (phase changes occurred at different instants of the loading cycle). In the second cycle, since the starting configuration is not homogeneous, the elements reach the instability condition one by one (see [3] for details) and during phase changes the elements in the low deformation phase do not have the same value of deformation. 
This effect is due to the viscous character of the response (the Maxwell elements) and it is magnified by damage, see Figure 13a.

In Figure 13b we compare the deformation of the elements at loading for $\varepsilon_{0}=0.45$ in the first cycle and in the second cycle (points $\mathrm{C}$ and $\mathrm{H}$ in Figure 11) with and without damage. Without damage, only small differences are observed between first and second cycle. The elements in the low deformation phase have the same deformation in the first cycle, while they take slightly different values of deformation in the second cycle. But in both cases, there are no elements with deformation values in the interval $[0.07,0.54]$, and there is the same number of elements in the two phases. As discussed in Section 3.3, damage enlarges the interval of the spring deformation values that satisfy the stability condition (12). For this reason, a different distribution of the total prescribed deformation on the damaged elements is observed in the second cycle in Figure 13b. Only few elements are in the low deformation phase and there exist elements with deformation values between 0.2 and 0.54 .

The differences between steps $\mathrm{C}$ and $\mathrm{H}$ in Figure 11 are therefore due to the interaction of viscosity and damage. Without viscosity, the system would return to the initial homogeneous configuration at the end of each loading-unloading cycle. During phase changes both at first and subsequent cycles the elements would be grouped into two blocks characterized by two distinct values of deformation. Without damage, the interval of deformation values in which the elements are stable would not change after the first cycle, so that the differences in the distribution of the deformations between the first and the subsequent cycles could not be distinguished.

The fact that in the model the localization of deformation occurs first at the bottom of the chain is an artifact due to the numerical scheme, because this model cannot predict where phase change will occur. A more detailed evolution of phase changes can be obtained by taking into account for non local effects with long-range linear springs, as shown in [25] in the case of shape memory alloys.

The construction of the model and the identification of the material constants were conducted on a series of basic cycles. In the following sections, we will test the ability of this model to describe the behavior of the foam under non standard loadings.

\subsection{Loading-unloading cycles with different amplitudes}

In Figure 14, the results of numerical simulations (left column) of three loading-unloading cycles with different amplitudes are compared with the corresponding experimental data (right column).

Note that in all three tests, the model predicts the significant strength reduction between the first and subsequent cycles observed in the experiments. In particular, one sees in Figure 14c that the simulation reproduces fine details such as the change in the curvature of the response curve at the second cycle for $\varepsilon_{0}=0.3$. This effect is due to the damage introduced on the non linear elastic springs. At the end of the first loading for $\varepsilon_{0}=0.4$, some springs are in the high deformation phase and are therefore damaged. In the subsequent cycles the damaged springs change phase for lower values of force, so that at $\varepsilon_{0}=0.3$ all the damaged springs have already changed phase and only undamaged springs are in the low deformation phase. At this point the change of phase is temporally stopped and the response curve rises up till the values of force necessary to start the change of phase of the undamaged springs are reached.

In all the simulation results the value of the force in the plateau at unloading is slightly underestimated. This discrepancy was foreseen as it was already observed during the identification of the material constants, see Figure 10.

As described in Section 3.3, we used a simple discrete evolution law for the damage parameter $d$, so that the elements have only two stages: undamaged $(d=0)$ or damaged $(d=\bar{d})$. The evolution of damage in the foam is therefore uniquely described by the number of damaged elements. When cycles of constant amplitude are applied, damage occurs only in the first loading process during the changes of phase of the springs. The amplitude of the cycle determine the number of elements that undergo damage and so the dimension of the damaged area. The convenient fit between theoretical and experimental results for various maximum deformation amplitudes is a first validation step of the model. 
(a)

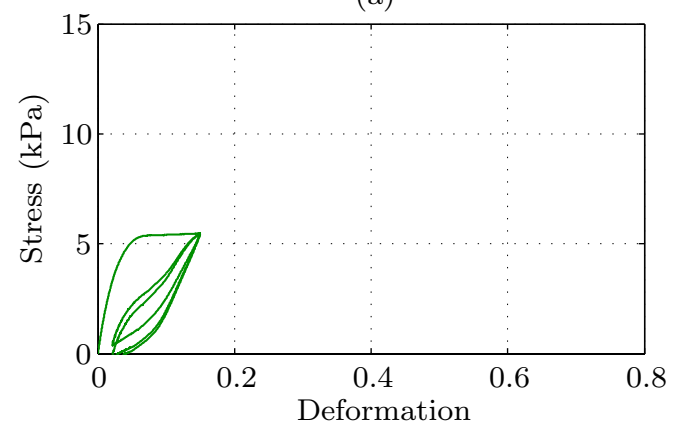

(c)

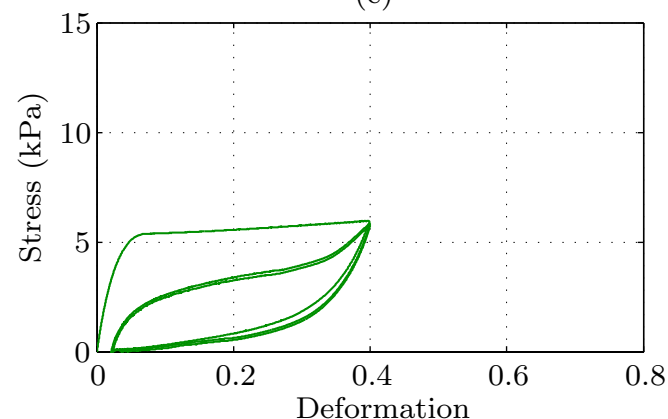

(e)

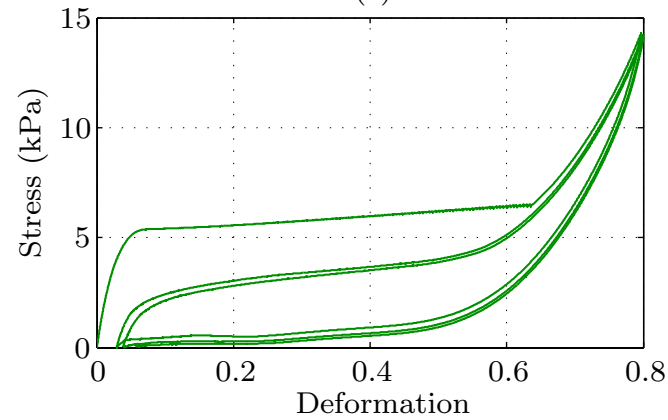

(b)

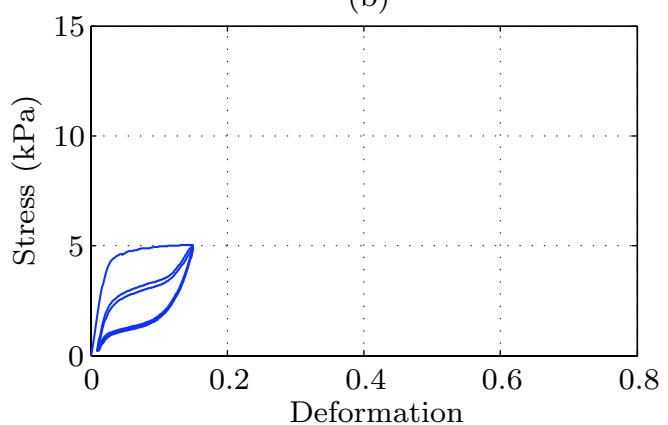

(d)

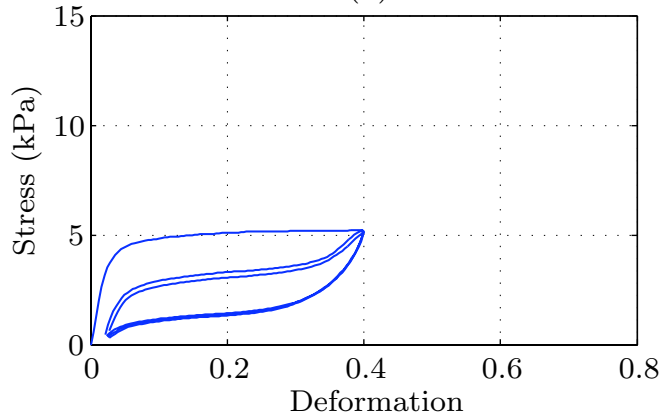

(f)

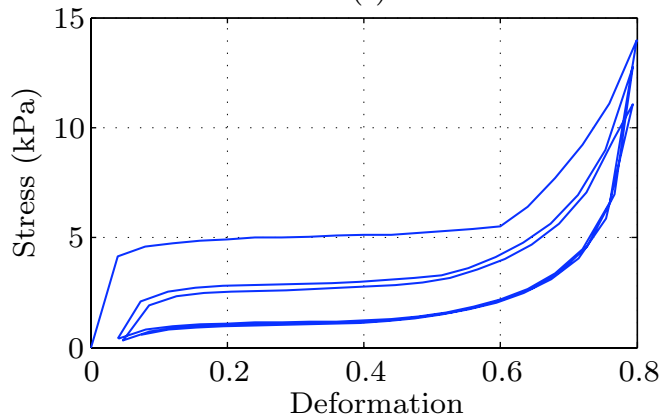

Fig. 14 Loading-unloading cycles with different amplitudes. Numerical simulations (a), (c), (e), and experiments (b), (d), (f).

6.3 Loading-unloading cycles with resting periods

The numerical simulations of the cyclic compression tests with two-day and 17-day resting period described in Section 2 are shown in Figure 15. It can be noted that the simulations capture the following features of the experimental response curves:

- the stress value $(4 \mathrm{kPa})$ in the plateau and the plateau length of the first loading cycle;

- the stress value $(8 \mathrm{kPa})$ reached at the end of the first loading process;

- the values of the residual deformation observed at the end of each loading-unloading cycle;

- the response curves obtained with 17-days resting period match those obtained after two days.

However, there is a major discrepancy between simulations and experiments in the response in the subsequent cycles. The curves at re-loading approach that obtained in the first cycle faster in the simulations than in the experiments. At $\varepsilon=0.4$, the stress difference between the first and second loading curve is about $0.2 \mathrm{kPa}$ in the simulations, while it is almost $1 \mathrm{kPa}$ in the experiments. This discrepancy, probably due to a restoring effect, needs to be investigated in more detail. 
(a)

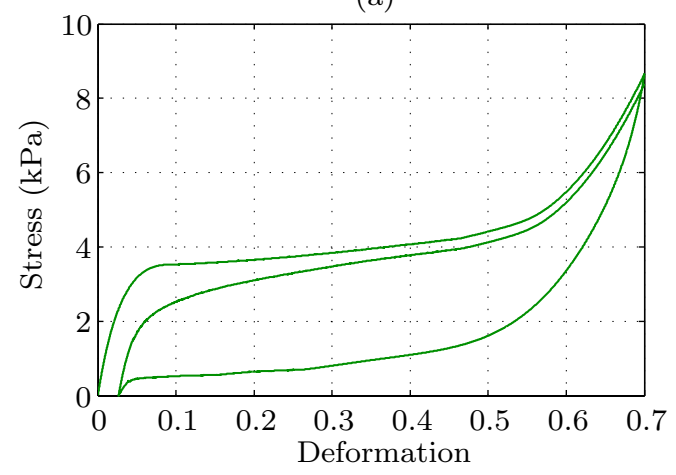

(c)

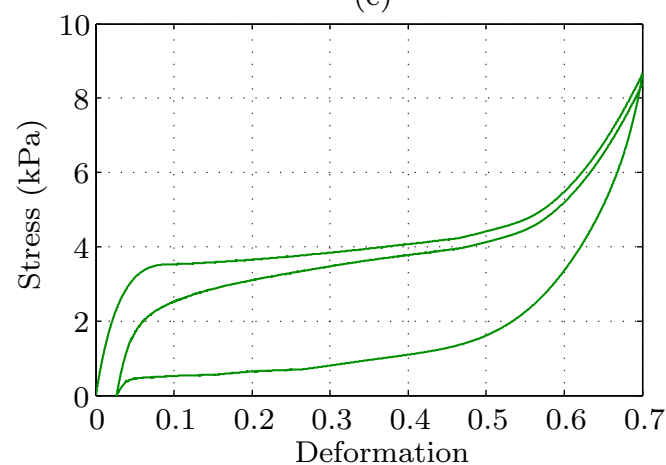

(b)

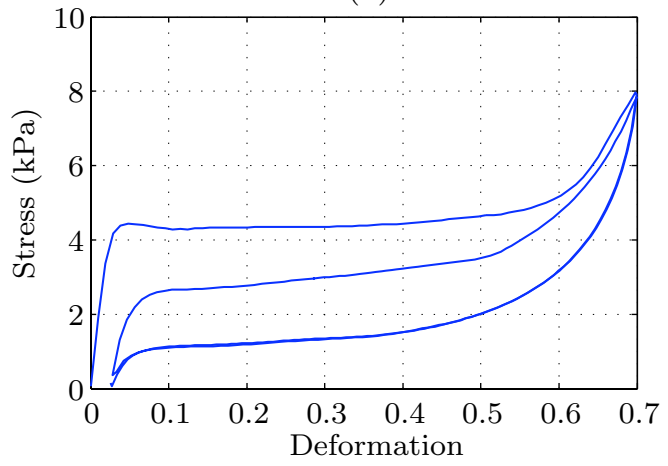

(d)

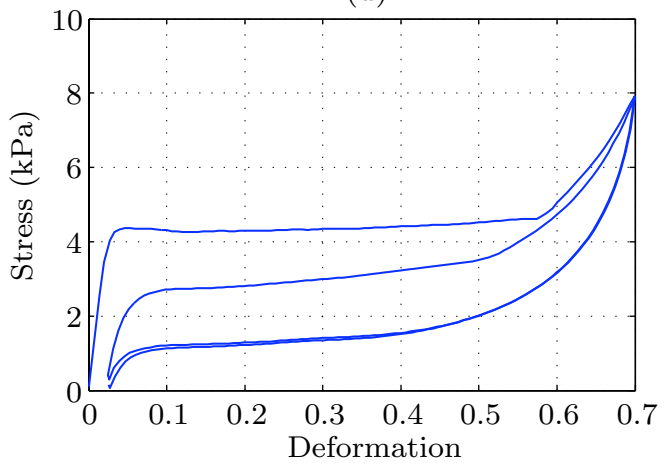

Fig. 15 Cyclic tests after a two-day resting period (a), (b) and a 17-day resting period (c), (d). Numerical simulations (a), (c), and experiments (b), (d).

\subsection{Complex loading-unloading cycles}

Complex tests with various combinations of loading, unloading, relaxation and resting periods were simulated. The results of the numerical simulations (on the left), and the experimental curves (on the right) are compared in Figure 16. All the simulations were conducted with the same set of parameters specified in Section 5 .

The first simulation (Figure 16a) consisted of two series of four loading-unloading cycles of different amplitudes separated by a resting period of $25 \mathrm{~s}$. In the first series of four cycles, some characteristics of the experimental curves cannot be found in the simulations: the plateau regimes almost disappear after the first cycle, and a considerable difference between the first unloading curve and the subsequent ones is observed. On the other hand, the response during the second series of four cycles is accurately simulated. The first loading curve shows two plateau regimes separated by an ascending branch, and all the unloading curves are very close to each other.

Two simulations of cyclic tests with different amplitudes including relaxation periods are shown in Figures 16c, 16e. Comparisons with the corresponding experimental data presented in the righthand column (Figures 16d, 16f) show that the model predicts the behavior of the foam subjected to complex loading processes. In particular:

- the shape of the hysteresis loops are reproduced in both large and small amplitude cycles;

- the last loading curve ends on the first loading curve;

- the small vertical segments corresponding to the relaxation periods are captured.

The model allows to clarify the role played by non-linear elasticity, viscosity and damage in the complex behavior of foam. For example in Figure 16 the small hysteresis loop and the small vertical segments are due to the viscous properties of the foam, the large hysteresis loops is described by the non convex strain energy density, and the change in shape of the loading curve as the number of cycle increases is due to damage. 
(a)

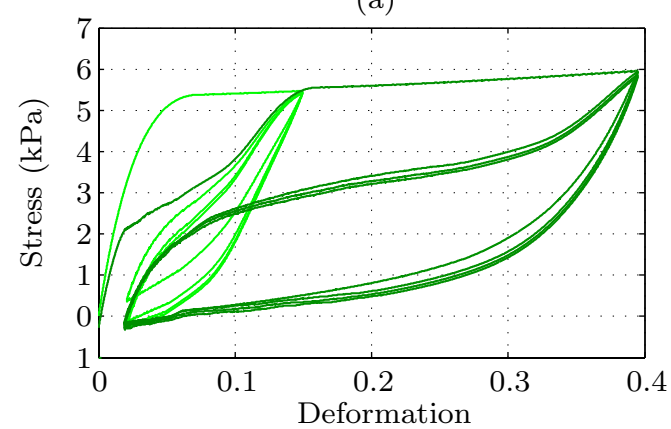

(c)

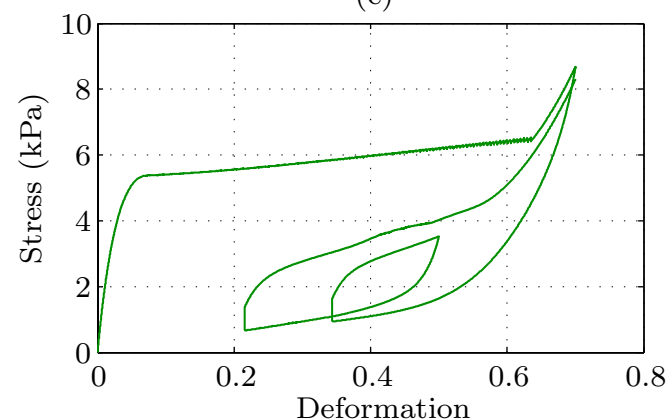

(e)

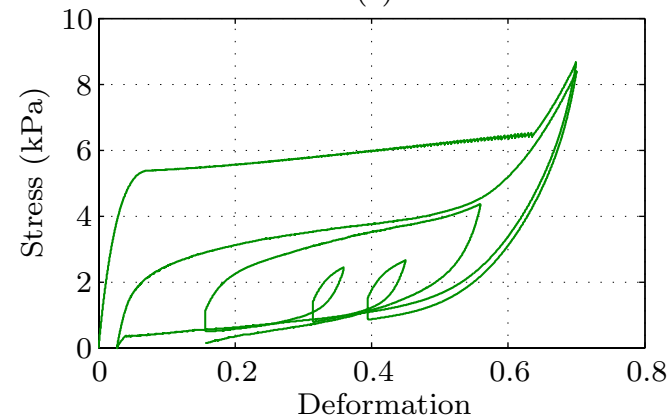

(b)

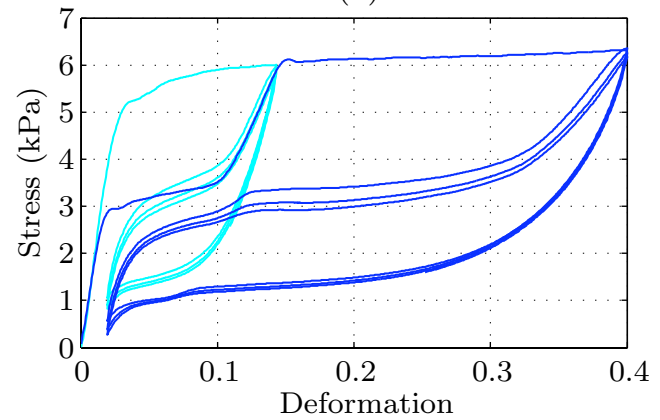

(d)

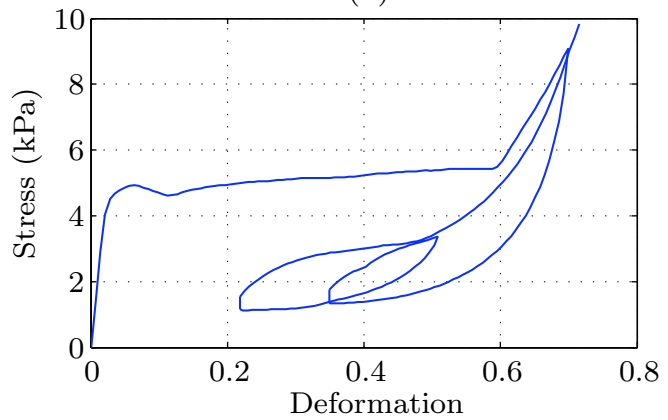

(f)

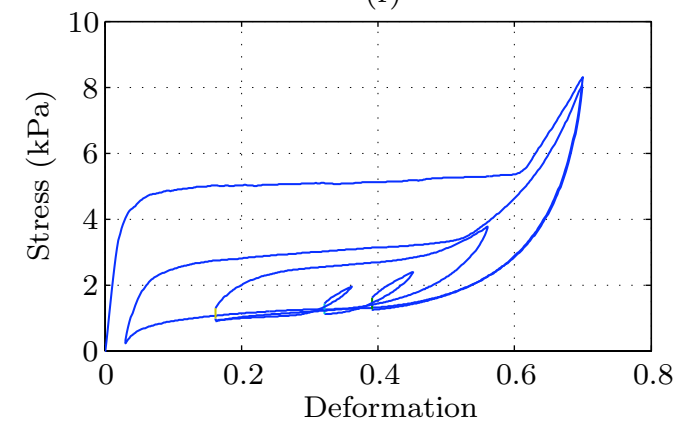

Fig. 16 Complex loading-unloading cycles. Numerical simulations (a), (c), (e), and experiments (b), (d), (f).

\section{Summary and conclusions}

In this paper the behavior of an open-cell polymer foam subjected to uniaxial compression cycles was studied. The analysis of key experimental tests show the importance to take into account for non linear elastic, viscous and damage effects to model the response of the foam.

The corresponding model is presented in this paper by extending the model by Del Piero and Pampolini [3] with introduction of damage. A special care has been devoted to the identification procedure for the material constants, and to the validation of the model on non standard loadings.

Of particular interest is the ability of the model to capture fine details of the foam behavior, as the response to complex loadings and the differences of the foam deformation evolutions between the first and the subsequent loading processes. In spite of its academic character (one dimensional, discrete, accounting only for linear viscous behavior), this model gives useful insights on how nonlinear elasticity, viscosity and damage can be combined to describe the behavior of open-cell foams.

The main perspective of this work will be to introduce non linearity on the viscosity.

Acknowledgements The work conducted by G. Pampolini during his joint Ph.D. project between the University of Provence and the University of Ferrara was supported by the Vinci grant awarded by the University Franco-Italienne. 


\section{References}

1. Bardenhagen, S.G., Brydon, A.D., Guilkey, J.E.: Insight into the physics of foam densification via numerical simulation. J. Mech. Phys. Solids 53, 597-617 (2005)

2. Beatty, M.F., Krishnaswamy, S.: A theory of stress-softening in incompressible isotropic materials. J. Mech. Phys. Solids 48, 1931-1965 (2000)

3. Del Piero, G., Pampolini, G.: The influence of viscosity on the response of open-cell polymeric foams in uniaxial compression: experiments and theoretical model. Continuum Mech. Thermodyn. 24, 181-199 (2012)

4. Diani, J., Fayolle, B., Gilormini, P.: A review on the Mullins effect. Eur. Polym. J. pp. 601-612 (2009)

5. Dorfmann, A., Ogden, R.W.: A pseudo-elastic model for loading, partial unloading and reloading of particlereinforced rubber. Int. J. Solids Struct. 40, 2699-2714 (2003)

6. Ericksen, J.L.: Equilibrium of bars. J. Elast. pp. 191-201 (1975)

7. Gibson, L.J., Ashby, M.F.: Cellular Solids: Structure and Properties, second edn. Cambridge University Press (1997)

8. Gioia, G., Wang, Y., Cuitiño, A.M.: The energetics of heterogeneous deformation in open-cell solid foams. Proc. R. Soc. Lond. A 457, 1079-1096 (2001)

9. Gong, L., Kyriakides, S.: Compressive response of open-cell foams. Part II: Initiation and evolution of crushing. Int. J. Solids Struct. 42, 1381-1399 (2005)

10. Gong, L., Kyriakides, S., Jang, W.Y.: Compressive response of open-cell foams. Part I: Morphology and elastic properties. Int. J. Solids Struct. 42, 1355-1379 (2005)

11. Gong, L., Kyriakides, S., Triantafyllidis, N.: On the stability of Kelvin cell foams under compressive loads. J. Mech. Phys. Solids 42, 1381-1399 (2005)

12. Gurtin, M., Francis, E.: Simple rate independent models for damage. J. Spacecraft 18, 285-286 (1981)

13. Holmström, K., Petersson, J.: A review of the parameter estimation problem of fitting positive exponential sums to empirical data. Appl. Math. Comput. pp. 31-61 (2002)

14. Hooke, R., Jeeves, T.A.: Direct search solution of numerical and statistical problems. J. Assoc. Computing Machinery 8, 212-229 (1960)

15. Jang, W.Y., Kraynik, A.M., Kyriakides, S.: On the microstructure of open-cell foams and its effect on elastic properties. Int. J. Solids Struct. 45, 1845-1875 (2008)

16. Kachanov, L.M.: Introduction to Continuum Damage Mechanics. Martinus Nijhoff Publishers (1986)

17. Ko, W.L.: Deformations of foamed elastomers. J. Cell. Plast. 1, 45-50 (1965)

18. Lemaitre, J., Chaboche, J.L.: Mechanics of Solid Materials. Cambridge University Press (1990)

19. Miehe, C., Keck, J.: Superimposed finite elastic-viscoelastic-plastoelastic stress response with damage in filled rubbery polymers. Experiments, modelling and algorithmic implementation. J. Mech. Phys. Solids 48, 323-365 (2000)

20. Mills, N.J.: The high strain mechanical response of the wet kelvin model for open-cell foams. Int. J. Solids Struct. 44, 51-65 (2007)

21. Mullins, L.: Softening of rubber by deformation. Rubber Chem. Technol. 42, 339-362 (1969)

22. Ogden, R.W., Roxburg, D.G.: A pseudo-elastic model for the Mullins effect in filled rubber. Proc. Roy. Soc. Lond. A 455, 2861-2877 (1999)

23. Pampolini, G.: Les propriétés mécaniques des mousses polymériques à cellules ouvertes: expériences, modéle théorique et simulations numériques. Ph.D. thesis, Laboratoire de Mécanique et Acoustique CNRS, Université de Provence, Università di Ferrara (2010)

24. Pampolini, G., Del Piero, G.: Strain localization in open-cell polyurethane foams: experiments and theoretical model. J. Mech. Mater. Struct 3, 969-981 (2008)

25. Puglisi, G.: Hysteresis in multi-stable lattices with non-local interactions. J. Mech. Phys. Solids 54, 2060-2088 (2006)

26. Raous, M.: Détermination du module d'Young opérationnel d'un corps viscoléastique à partir de sa courbe de relaxation. Rheologica Acta 13, 1233-1237 (1974)

27. Romero, P.A., Zheng, S.F., Cuitiño, A.M.: Modeling the dynamic response of visco-elastic open-cell foams. J. Mech. Phys. Solids 58, 1916-1943 (2008)

28. Simo, J.: On a fully three dimensional finite strain viscoelastic damage model: Formulation and computation aspects. Comput. Mech. Appl. Mech. Engrg. 60, 153-173 (1987)

29. Sorrentino, L., Aurelia, M., Iannace, S.: A simple method to predict high strain rates mechanical behavior of low interconnected cell foams. Polymer Testing 26, 878-885 (2000)

30. Sullivan, R.M., Ghosn, L.J., Lerch, B.A.: A general tetrakaidecahedron model for open-celled foams. Int. J. Solids Struct. 45, 1754-1765 (2008)

31. Viot, P., Iordanoff, I., Bernard, D.: Multiscale description of polymeric foam behavior: a new approach based on discrete element modeling. Polym. Sci. (50), 679-689 (2008)

32. Wang, Y., Cuitiño, A.M.: Full-field measurements of heterogeneous deformation patterns on polymeric foams using digital image correlation. Int. J. Solids Struct. 39, 3777-3796 (2002)

33. Warren, W., Kraynik, A.M.: The non linear elastic properties of open-cell foams. ASME J. Appl. Mech. 58, 376-381 (1991)

34. White, S., Kim, S., Bajaj, A., Davis, P.: Experimental techniques and identification of nonlinear and viscoelastic properties of flexible polyurethane foam. Nonlinear Dynamics 22, 281-313 (2000) 